\title{
Properties of Multigranular Rough Sets on Fuzzy Approximation Spaces and their Application to Rainfall Prediction
}

\author{
B.K. Tripathy \\ VIT University, SCOPE, Vellore-632014, INDIA \\ E-mail: tripathybk@vit.ac.in \\ Urmi Bhambhani \\ VIT University, SCOPE, Vellore-632014, INDIA \\ E-mail: urmibhambhani@gmail.com
}

Received: 30 June 2017; Accepted: 11 November 2017; Published: 08 November 2018

\begin{abstract}
Basic rough set model introduced by Pawlak in 1982 has been extended in many directions to enhance their modeling power. One such attempt is the notion of rough sets on fuzzy approximation spaces by De et al in 1999. This basic model uses equivalence relation for its definition, which decompose the universal set into disjoint equivalence classes. These equivalence classes are called granules of knowledge. From the granular computing point of view the basic rough set model is unigranular in character. So, in order to handle more than one granular structure simultaneously, two types of multigranular rough sets, called the optimistic and pessimistic multigranular rough sets were introduced by Qian et al in 2006 and 2010 respectively. In this paper, we introduce two types of multigranular rough sets on fuzzy approximation spaces (optimistic and pessimistic), study several of their properties and illustrate how this notion can be used for prediction of rainfall. The introduced notions are explained through several examples.
\end{abstract}

Index Terms-Rough sets, multigranulation, fuzzy approximation space, rain prediction.

\section{INTRODUCTION}

Several models have been put forth in order to handle uncertainty in data. The notion of fuzzy sets introduced by Zadeh [21] and the notion of rough sets introduced by Pawlak [41, 42] are two of the most fruitful models so far. The notion of rough sets is based on the philosophy that human knowledge is dependent upon their classificatory capabilities. As a consequence the mathematical notion of equivalence relations is used in defining rough sets by Pawlak, which induce classifications on the universes over which they are defined. However, the relative scarcity of equivalence relations led to the relaxation of the requirements of this notion to develop rough sets based upon proximity relations [1] which is equivalence relations minus the transitivity condition and similarity relations which are supposed to be only reflexive [28]. Fuzzy relations were introduced as extensions of the crisp relations to define the relations among elements in a more natural way. The notions of fuzzy equivalence relations and that of the fuzzy proximity relations are special kinds of fuzzy relations. A new type of rough set was defined by De et al [29] basing upon fuzzy proximity relations, which is called rough sets on fuzzy approximation spaces. These rough sets depend upon an indexing variable lying in the interval $[0,1]$. Some properties of such rough sets are derived in $[9,10,17]$.

The notion of granular computing in the context of fuzzy sets was introduced by Zadeh [22, 23] and also he was responsible for defining this notion into the realm of rough sets [24]. However, the name granular computing was coined by T.Y.Lin [30]. Granular computing has been strongly encouraged by the need for processing practical data in an intelligent manner [25, 31, 32, 33]. Such processing need is now commonly available in vast quantities into a humanly manageable abstract knowledge. In other words, granular computing offers a platform for transition from the current machine-centric to humancentric approach to gather information and knowledge. Granular computing as opposed to numeric computing is knowledge oriented. Generally speaking, information granules are collections of entities that usually arranges together due to their similarity, functional or physical adjacency, indistinguishability etc. In other words information granulation involves partitioning a class of objects into granules with a granule being a bunch of objects which are drawn together by indistinguishability, similarity or functionality. The branch of granular computing has been explored extensively by Yao [3640].From the granular computing point of view the rough set notions introduced above were unigranular; that is only one relation, equivalence or otherwise was being used at a time to define such rough sets. However, single granular structure cannot always be satisfied in many practical issues. For instance, it cannot be used to deal 
with distribution data; it is very time-consuming for feature selection in big data set $[19,20]$. As a consequence in 2006 the first type of multigranulation, called the optimistic multigranular rough sets was introduced by Qian et al [34]. The second type of multigranulation called the pessimistic multigranular rough sets was introduced by Qian et al [35]. Several properties of these two types of multigranulation and their extensions have been established over the past few years $[6,7,8,12,13,16,26]$.

In this paper, we define the notion of multigranular rough sets on fuzzy approximation spaces, which are extension models of the basic multigranular models, study their properties and discuss on their applications. The further organization of the chapter is as follows. In the second section, we present the definitions and notations to be used throughout this work and prove a few elementary results on rough sets on fuzzy approximation spaces. In the third section, we introduce both the notions of optimistic and pessimistic multigranular rough sets on fuzzy approximation spaces. In the fourth section, we establish several properties of the two new notions. In the following section, we present some applications of these concepts and in the final section, the concluding remarks are provided. This is followed by the bibliography of papers referred during the compilation of the work.

\section{RELATED WORKS}

The notion of rough sets on fuzzy approximation spaces introduced by De et al [29] was extended to define the generalised notion of rough sets on intuitionistic fuzzy approximation spaces by Tripathy [2]. Many properties of these spaces can be found in [3]. As mentioned by Pawlak [42] one of the two properties of rough sets for any of its fruitful application is topological characterization. The other property is being the accuracy measure. Many properties of rough sets basing upon their topological characteristic can be found in the works of B. $\mathrm{K}$. Tripathy $[4,14]$. The notion of equality of sets used in mathematics is too stringent in the sense that the sets comprise of the same collection of elements with different levels. In real life situations, we use a kind of approximate equality of sets. It involves the knowledge of the observer. Basing upon this observation, the notions of rough set based equalities were introduced by Pawlak in [42]. These concepts have been extended to the context of multigranular rough sets as can be found in [11, 15, 27]. A study of topological properties of these models can be found in [5, 14]. In this article we study some more properties of multigranular rough sets on fuzzy approximation spaces.

\section{DEFINITIONS AND NOTATIONS}

In this section we shall introduce some definitions and notations which are to be used in this paper. As mentioned in the introduction, fuzzy set is one of the most successful imprecise models introduced by Zadeh [21]. It is defined as follows.

Definition 1: Let $U$ be a universal set. Then a fuzzy subset $X$ of $U$ is defined through a function $\mu_{X}$, called the membership function of $X$ such that $\mu_{X}: U \rightarrow[0,1]$, which assigns grades of membership values to elements of $U$ into $X$ lying in the interval $[0,1]$.

The notion of rough set introduced by Pawlak [41] was supposed to be a competitor for fuzzy sets after its inception was actually found to complement it. It follows the boundary region approach in capturing uncertainty of belongingness of elements into a set introduced by Frege. We first need the following set up in order to formally define it.

Let $R$ be an equivalence relation over $U$. By $U / R$ we denote the family of all equivalence classes of $R$, referred to as categories or concepts of $\mathrm{R}$ and the equivalence class of an element $x \in U$ is denoted by $[x]_{R}$. By a knowledge base, we understand a relational system $\mathrm{K}$ $=(\mathrm{U}, \mathrm{P})$, where $\mathrm{U}$ is as above and $\mathrm{P}$ is a family of equivalence relations over $\mathrm{U}$. For any subset $Q(\neq \phi) \subseteq P$, the intersection of all equivalence relations in $\mathrm{Q}$ is denoted by $\operatorname{IND}(\mathrm{Q})$ and is called the indiscernibility relation over $\mathrm{Q}$. Let us define $\operatorname{IND}(\mathrm{K})=\{\operatorname{IND}(\mathrm{Q})$ $Q \subseteq P\}$

Definition 2: Given any $X \subseteq U$ and $R \in I N D(K)$ we associate two subsets $\underline{R} X$ and $\bar{R} X$, called the R-lower and $\mathrm{R}$-upper approximations of $\mathrm{X}$ respectively, defined by

$$
\begin{aligned}
& \underline{R} X=\left\{x \in U \mid[x]_{R} \subseteq X\right\} \\
& \bar{R} X=\left\{x \in U \mid[x]_{R} \cap X \neq \phi\right\}
\end{aligned}
$$

$\mathrm{X}$ is said to be rough with respect to $\mathrm{R}$ if and only if $\underline{R} X \neq \bar{R} X$ and R-definable otherwise. The R-boundary of $\mathrm{X}$ is denoted by $B N_{R}(X)$ and is defined as $B N_{R}(X)=\bar{R} X \backslash \underline{R} X$. Clearly $\mathrm{X}$ is R-definable if and only if $B N_{R}(X)=\phi$ and rough otherwise.

The elements of $\underline{R} X$ are those elements of $\mathrm{U}$, which can certainly be classified as elements of $X$, and the elements of $\bar{R} X$ are those elements of $\mathrm{U}$, which can possibly be classified as elements of $\mathrm{X}$, employing knowledge of R.

A relation over $U$ which is only reflexive and symmetric is called a proximity relation. A fuzzy relation over $\mathrm{U}$ is a fuzzy subset of $U \times U$. For any fuzzy relation with membership function $\mu_{R}$ we have the following.

Definition 3: For any fuzzy relation R over U

$\mathrm{R}$ is fuzzy reflexive if and only if $\mu_{R}(x, x)=1, \forall x \in U$. 


$$
\begin{gathered}
\mathrm{R} \text { is fuzzy symmetric if and only if } \\
\mu_{R}(x, y)=\mu_{R}(y, x), \forall x, y \in U .
\end{gathered}
$$

A fuzzy relation $\mathrm{R}$ is said to be a fuzzy proximity relation if and only if $\mathrm{R}$ is both fuzzy reflexive and fuzzy symmetric.

Definition 4: For any $\alpha \in[0,1]$, the $\alpha$-cut of $\mathrm{R}$ is denoted by $R_{\alpha}$ is a subset of $U \times U$ is given by

$$
R_{\alpha}=\left\{(x, y) \mid \mu_{R}(x, y) \geq \alpha\right\} .
$$

For any fuzzy proximity relation $\mathrm{R}$ on $\mathrm{U}$ and $\alpha \in[0,1]$, if $(x, y) \in R_{\alpha}$ then we say that $\mathrm{x}$ and $\mathrm{y}$ are $\alpha$-similar and we denote it by $x R_{\alpha} y$.

Definition 5: Two elements $\mathrm{x}$ and $\mathrm{y}$ are said to be $\alpha$-identical denoted by $x R(\alpha) y$ if either $x R_{\alpha} y$ or there exists a sequence of elements $u_{1}, u_{2}, \ldots u_{n}$ in $\mathrm{U}$ such that $x R_{\alpha} u_{1} R_{\alpha} u_{2} \ldots u_{n} R_{\alpha} y$.

It may be noted that the relation $R(\alpha)$ is an equivalence relation for each $\alpha \in[0,1]$. Here (U, R) is called a fuzzy approximation space. Also, it may be noted that for any $\alpha \in[0,1],(U, R(\alpha))$ is an approximation space in the same sense as that used by Pawlak.

For any $\mathrm{x}$ in $\mathrm{U}$ we denote the equivalence class of $\mathrm{x}$ with respect to $R(\alpha)$ by $[x]_{R(\alpha)}$.

Definition 6: Let $U$ be a universal set and $R$ be a fuzzy proximity relation on $\mathrm{U}$. Then for any $\alpha \in[0,1]$ we define the lower and upper approximations of a subset $\mathrm{X}$ in $\mathrm{U}$ as

$$
\begin{gathered}
\qquad \frac{R(\alpha) X}{}=\left\{x \in U \mid[x]_{R(\alpha)} \subseteq X\right\} \\
\text { and } \overline{R(\alpha)} X=\left\{x \in U \mid[x]_{R(\alpha)} \cap X \neq \phi\right\} .
\end{gathered}
$$

We say that $\mathrm{X}$ is $R(\alpha)$ discernible if and only if $R(\alpha) X=\overline{R(\alpha)} X$. Else, $\mathrm{X}$ is said to be $R(\alpha)$-rough.

Several properties of rough sets on fuzzy approximation spaces have been considered in $[3,7,9$, 10]. We present below some more definitions and results on rough sets on fuzzy proximity relations which are necessary in the work done in this paper.

Definition 7: For any two fuzzy relations $\mathrm{R}$ and $\mathrm{S}$ on $\mathrm{U}$ we define their union and intersection $R \cup S$ and $R \cap S$ as follows:

$$
\begin{aligned}
& \mu_{(R \cup S)}(x, y)=\max \left\{\mu_{R}(x, y), \mu_{S}(x, y)\right\} \\
& \mu_{(R \cap S)}(x, y)=\min \left\{\mu_{R}(x, y), \mu_{S}(x, y)\right\}
\end{aligned}
$$

Note 1: It may be noted that with the above definitions, $R \cup S$ and $R \cap S$ are fuzzy proximity relations on $\mathrm{X}$ when $\mathrm{R}$ and $\mathrm{S}$ are so. Hence $(R \cup S)_{\alpha},(R \cap S)_{\alpha}$ and as a consequence $(R \bigcup S)(\alpha),(R \cap S)(\alpha)$ are meaningful.

Lemma 1: For any two fuzzy proximity relations $\mathrm{R}$ and $\mathrm{S}$ on $\mathrm{U}$ and any $\alpha \in[0,1]$, we have

$$
\begin{gathered}
(R \cup S)_{\alpha}=R_{\alpha} \cup S_{\alpha} \\
\text { and }(R \cap S)_{\alpha}=R_{\alpha} \cap S_{\alpha}
\end{gathered}
$$

Proof: Proof of (10): We have

$$
\begin{aligned}
(x, y) \in(R \cup S)_{\alpha} & \Leftrightarrow \mu_{(R \cup S)}(x, y) \geq \alpha \\
& \Leftrightarrow \max \left\{\mu_{R}(x, y), \mu_{S}(x, y)\right\} \geq \alpha \\
& \left.\Leftrightarrow \mu_{R}(x, y) \geq \alpha \text { or } \mu_{S}(x, y)\right\} \geq \alpha \\
& \Leftrightarrow(x, y) \in R_{\alpha} \text { or }(x, y) \in S_{\alpha} \\
& \Leftrightarrow(x, y) \in R_{\alpha} \cup S_{\alpha}
\end{aligned}
$$

Similarly (11) can be proved.

Let $R_{\alpha}^{*}, S_{\alpha}^{*},(R \cup S)_{\alpha}^{*}$ and $(R \cap S)_{\alpha}^{*}$ denote the set of equivalence classes generated by $R(\alpha), S(\alpha)$, $(R \cup S)(\alpha)$ and $(R \cap S)(\alpha)$ respectively.

Then it was shown in [17] that the results established by De et al in [29] in the following form are incorrect.

Result1: For any $\alpha \in[0,1]$,

(i) $\quad(R \bigcup S)(\alpha) \subseteq R(\alpha) \bigcup S(\alpha)$

(ii) $(R \cap S)(\alpha) \supseteq R(\alpha) \cap S(\alpha)$

The correct result as shown in [10] is as follows.

Lemma 2: For any $\alpha \in[0,1]$,

(i) $\quad(R \bigcup S)(\alpha) \supseteq R(\alpha) \bigcup S(\alpha)$

(ii) $(R \cap S)(\alpha) \subseteq R(\alpha) \cap S(\alpha)$

Note 2: The results in Lemma 2.1 are sometimes more useful in the following forms.

For any $\alpha \in[0,1]$ and $x \in U$, we have

$$
\begin{gathered}
{[x]_{R(\alpha)} \cup[x]_{S(\alpha)} \subseteq[x]_{R(\alpha) \cup S(\alpha)}} \\
\text { And }[x]_{R(\alpha) \cap S(\alpha)} \subseteq[x]_{R(\alpha)} \cap[x]_{S(\alpha)}
\end{gathered}
$$

Next, we prove a result which establishes the relations between the lower, upper approximations of a set $\mathrm{X}$ with respect to $(R \cup S)(\alpha),(R \cap S)(\alpha)$ and the union and intersection of its lower and upper intersections of $R(\alpha)$ and $S(\alpha)$ respectively. 
Theorem 1: Let $\mathrm{R}$ and $\mathrm{S}$ be two fuzzy proximity relations on $\mathrm{U}$ and $\alpha$ be a chosen level value. Then

$$
\begin{aligned}
& \underline{(R \cup S)(\alpha)} X \subseteq \underline{R(\alpha)} X \cup \underline{S(\alpha)} X \\
& \overline{(R \bigcup S)(\alpha)} X \supseteq \overline{R(\alpha)} X \cup \overline{S(\alpha)} X \\
& \underline{(R \cap S)(\alpha)} X \supseteq \underline{R(\alpha)} X \cap \underline{S(\alpha) X} \\
& \overline{(R \cap S)(\alpha)} X \subseteq \overline{R(\alpha)} X \cap \overline{S(\alpha)} X
\end{aligned}
$$

Proof: Proof of (14): We have

$$
\begin{gathered}
\frac{(R \bigcup S)(\alpha) X}{}=\left\{x \mid[x]_{(R \cup S)(\alpha)} \subseteq X\right\} \\
\subseteq\left\{x \mid[x]_{R(\alpha)} \cup[x]_{S(\alpha)} \subseteq X\right\} \\
\subseteq\left\{x \mid[x]_{R(\alpha)} \subseteq X\right\} \bigcup\left\{x \mid[x]_{S(\alpha)} \subseteq X\right\} \\
=\underline{R(\alpha) X \bigcup \underline{S(\alpha) X}}
\end{gathered}
$$

This proves (14). Proof of (15) is similar. Proof of (16): We have

$$
\begin{aligned}
& \underline{R(\alpha)} X \cap \underline{S(\alpha)} X=\left\{x \mid[x]_{R(\alpha)} \subseteq X\right\} \cap\left\{x \mid[x]_{S(\alpha)} \subseteq X\right\} \\
& =\left\{x \mid[x]_{R(\alpha)} \cap[x]_{S(\alpha)} \subseteq X\right\} \\
& \subseteq\left\{x \mid[x]_{R(\alpha) \cap S(\alpha)} \subseteq X\right\} \quad(b y(13)) \\
& \subseteq\left\{x \mid[x]_{(R \cap S)(\alpha)} \subseteq X\right\} \\
& =(R \cap S)(\alpha) X
\end{aligned}
$$

This proves (16). Proof of (17) is similar.

As mentioned above two types of multigranulations exist in literature. We introduce these two types below.

Definition 8: Let $\mathrm{K}=(\mathrm{U}, \mathbf{R})$ be knowledge base, $\mathbf{R}$ be a family of equivalence relations, $\mathrm{X} \subseteq \mathrm{U}$ and $R, S \in \mathbf{R}$. We define the optimistic multigranular [33] lower approximation and optimistic multigranular upper approximation of $\mathrm{X}$ with respect to $\mathrm{R}$ and $\mathrm{S}$ in $\mathrm{U}$ as

$$
\begin{gathered}
\underline{R+S} \mathrm{X}=\left\{\mathrm{x} \mid[\mathrm{x}]_{\mathrm{R}} \subseteq \mathrm{X} \text { or }[\mathrm{x}]_{\mathrm{S}} \subseteq \mathrm{X}\right\} \\
\text { and } \overline{R+S} \mathrm{X}=\sim(\underline{R+S}(\sim X)) .
\end{gathered}
$$

Definition 9: Let $\mathrm{K}=(\mathrm{U}, \mathbf{R})$ be a knowledge base, $\mathbf{R}$ be a family of equivalence relations, $\mathrm{X} \subseteq \mathrm{U}$ and $R, S \in \mathbf{R}$. We define the pessimistic multigranular [34] lower approximation and pessimistic multigranular upper approximation of $\mathrm{X}$ with respect to $\mathrm{R}$ and $\mathrm{S}$ in $\mathrm{U}$ as

$$
\begin{gathered}
\underline{R * S} \mathrm{X}=\left\{\mathrm{x} \mid[\mathrm{x}]_{\mathrm{R}} \subseteq \mathrm{X} \text { and }[\mathrm{x}]_{\mathrm{S}} \subseteq \mathrm{X}\right\} \\
\text { and } \overline{R * S} \mathrm{X}=\sim(\underline{R * S}(\sim X)) .
\end{gathered}
$$

Example 1: Let us provide some examples below to show the computation and physical meaning of the above concepts in real life situations.
Example 1.1: Let us consider all the cattle in a locality as our universe $\mathrm{U}$. We define an equivalence relation $\mathrm{R}$ over $\mathrm{U}$ by $\mathrm{x} R \mathrm{R}$ iff $\mathrm{x}$ and $\mathrm{y}$ are cattle of the same category. For definiteness let $U=\{$ Cow, Buffalo, Goat, Sheep, Bullock\}. The equivalence classes of $\mathrm{U}$ with respect to $\mathrm{R}$ are $\{\{$ Cow, Buffalo, Bullock $\},\{$ Goat, Sheep $\}$. We define another equivalence relation $\mathrm{S}$ as $\mathrm{x} \mathrm{S}$ y if and only if $x$ and $y$ are of the same size. We get three equivalence classes with respect to $\mathrm{S}$ as $\mathrm{U}=\{$ Small, Middle, Large $\}$. These are defined as Large $=\{$ Buffalo, Bullock $\}$, Middle $=\{$ Cow $\}$ and Small $=\{$ Goat, Sheep $\}$.

Then for any subset $\mathrm{X}$ of the cattle in the society, we have,

$\underline{R+S} X$ is the set of cattle whose category is completely in $\mathrm{X}$ or all the cattle of its size are contained in $\mathrm{X}$.

$R * S X$ is the set of cattle whose category is completely in $\mathrm{X}$ and all the cattle of its size are contained in $\mathrm{X}$.

$\overline{R+S} X$ is the set of cattle some elements of whose category are in $X$ and some elements of whose size are in $\mathrm{X}$.

$\overline{R * S} X$ is the set of cattle some of whose category elements are in $\mathrm{X}$ or some elements of whose are in $\mathrm{X}$.

For example if we take $X=\{$ Cow, Bullock $\}$, then

$$
\begin{aligned}
& \underline{R+S} X=\{\text { Cow }\}, \overline{R+S} X=\{\text { Cow }, \text { Bufallo, Bullock }\} \\
& \underline{R * S} X=\phi, \overline{R * S} X=\{\text { Cow, Bufallo, Bullock }\}
\end{aligned}
$$

\section{Example 1.2: (Toy Example of Pawlak)}

Let us consider the example of toys taken by Pawlak [42] in order to explain the concepts of rough sets. Here, we have $U=\left\{x_{1}, x_{2}, x_{3}, x_{4}, x_{5}, x_{6}, x_{7}, x_{8}\right\}$ is the universe of 8 toys. These toys are of 3 different colours (red, blue and yellow), three different shapes (round, square and triangular) and two different sizes (large and small). The equivalence classes of the toys with respect to the relations; 'same colour as (R)', 'same shape as (S)' and 'same size as (T)' are given as follows:

$$
\text { Colour }=\left\{\left\{x_{1}, x_{3}, x_{7}\right\},\left\{x_{2}, x_{4}\right\},\left\{x_{5}, x_{6}, x_{8}\right\}\right\}
$$

Shape $=\left\{\left\{x_{1}, x_{5}\right\},\left\{x_{2}, x_{6}\right\},\left\{x_{3}, x_{4}, x_{7}, x_{8}\right\}\right\}$ and

Size $=\left\{\left\{x_{2}, x_{7}, x_{8}\right\},\left\{x_{1}, x_{3}, x_{4}, x_{5}, x_{6}\right\}\right\}$. Let us take a set $\mathrm{X}$ of toys as $\left\{x_{2}, x_{3}, x_{4}, x_{5}, x_{6}\right\}$.

Then applying the above definitions of multigranular lower and upper approximations we get

$\underline{R+S} X=\left\{x_{2}, x_{4}, x_{6}\right\}=$ The set of toys from $\mathrm{U}$ such that all toys of the same colour are in $X$ or all toys of the same shape are in $\mathrm{X}$.

$\overline{R+S} X=U=$ The set of toys from $\mathrm{U}$ at least one toy of the same colour is in $X$ and at least one toy of the same shape is in $X$. 
$\underline{R * S} X=\phi=$ The set of toys from $\mathrm{U}$ such that all toys of the same colour and all toys of the same shape are in X.

$\overline{R * S} X=U=$ The set of toys from $\mathrm{U}$ such that at least one toy of same colour or at least one toy of the same shape is in X.

The following properties of Multigranular rough sets shall be used by us. We consider the setting as in the above definitions.

Property 1: ([34]) We have

$$
\begin{aligned}
& \underline{R+S}(X \cap Y) \subseteq \underline{R+S}(X) \cap \underline{R+S}(Y) \\
& \underline{R+S}(X \cup Y) \supseteq \underline{R+S}(X) \cup \underline{R+S}(Y) \\
& \overline{R+S}(X \bigcup Y) \supseteq \overline{R+S}(X) \cup \overline{R+S}(Y) \\
& \overline{R+S}(X \cap Y) \subseteq \overline{R+S}(X) \cap \overline{R+S}(Y)
\end{aligned}
$$

Property 2: [35] We have

$$
\begin{aligned}
& \underline{R * S}(X \cap Y) \subseteq \underline{R * S}(X) \cap \underline{R * S}(Y) \\
& \underline{R * S}(X \cup Y) \supseteq \underline{R * S}(X) \cup \underline{R * S}(Y) \\
& \overline{R * S}(X \bigcup Y) \supseteq \overline{R * S}(X) \cup \overline{R * S}(Y) \\
& \overline{R * S}(X \cap Y) \subseteq \overline{R * S}(X) \cap \overline{R * S}(Y)
\end{aligned}
$$

Note 3: It was shown by Tripathy [9] that inclusion relations in (22) to (25) and (28) and (29) cannot be replaced with equalities.

Note 4: It was shown by Tripathy [9] that equality holds in (26) and (27).

It is interesting to find the case under which the two types of multigranulations reduce to single granulations. The following result was established by Tripathy et al in [9].

Property 3: Let $\mathrm{R}$ and $\mathrm{S}$ be two equivalence relations on $\mathrm{U}$ and $X \subseteq U$. Then

$$
\underline{R+S} X=\underline{R} X \text { and } \overline{R+S} X=\bar{R} X \text { when } S=U \times U
$$

$\underline{R * S} X=\underline{R} X$ and $\overline{R * S} X=\bar{R} X$ when $\mathrm{S}=\{(\mathrm{x}, \mathrm{x}) \mid x \in U\}$

Property 4: Let $\mathrm{R}$ and $\mathrm{S}$ be two equivalence relations on $\mathrm{U}$ and $X \subseteq U$. Then

$\underline{R * S} X \subseteq \underline{R+S} X \subseteq \underline{R \cap S} X \subseteq \overline{R \cap S} X \subseteq \overline{R+S} X \subseteq \overline{R * S} X$

All these inclusions in (32) can be strict. So as a consequence we derive that

$$
B N(R \cap S) \subseteq B N(R+S) \subseteq B N(R * S)
$$

Note 5: It is worth noting that the two operations ' + ' (optimistic multigranulation) and ' $*$ ' (pessimistic multigranulation) are commutative and associative. Hence, whatever result is true for 2 terms is also true for any finite number of components. So, throughout this paper we have considered only binary multigranulations, $\mathrm{n}$-ary multigranulations follow as a consequence.

Note 6: We also note that Raghavan et al [27] has used the notations (' + ' for two components and $\Sigma$ for more than two components) for the optimistic multigranulations and the notations ( ' * ' for two components and $\Pi$ for more than two components) for the pessimistic multigranulations instead of the conventional notations used in other papers. These notations are much simpler to use and look at.

As mentioned by Pawlak [42] any fruitful application of rough set theory should focus on the two concepts of accuracy measure and topological characterisation. Following this some characterisation of topological properties of multigranular rough sets have been established by Tripathy et al $[4,5,6]$.

\section{Multigranular Rough Sets On FuZZY APPROXIMATION SPACES}

In this section we introduce the concepts of optimistic multigranular rough sets and pessimistic multigranular rough sets on fuzzy approximation spaces.

We take $\mathrm{U}$ as a universal set and let $\mathrm{R}$ and $\mathrm{S}$ be two fuzzy proximity relations defined over $\mathrm{U}$. Then for any $\alpha \in[0,1]$, we define

$$
\begin{gathered}
\frac{R(\alpha)+S(\alpha) X}{}=\left\{x \in U:[x]_{R(\alpha)} \subseteq X \text { or }[x]_{S(\alpha)} \subseteq X\right\} \\
\overline{R(\alpha)+S(\alpha)} X \\
=\left\{x \in U:[x]_{R(\alpha)} \cap X \neq \phi \text { and }[x]_{S(\alpha)} \cap X \neq \phi\right\}
\end{gathered}
$$

Definition 10: A subset $X$ of $U$ is said to be $\operatorname{an}(\mathrm{R}+\mathrm{S})(\alpha)$-multigranular rough set if and only if $R(\alpha)+S(\alpha) X \neq \overline{R(\alpha)+S(\alpha)} X$. Otherwise, we say $\mathrm{X}$ is $(\mathrm{R}+\mathrm{S})(\alpha)$-multigranular definable.

We do not use the word optimistic in the definition as it is clear from the notation. We define,

$$
\underline{R(\alpha) * S(\alpha)} X=\left\{x \in U:[x]_{R(\alpha)} \subseteq X \text { and }[x]_{S(\alpha)} \subseteq X\right\}
$$

$\overline{R(\alpha) * S(\alpha)} X=\left\{x \in U:[x]_{R(\alpha)} \cap X \neq \phi\right.$ or $\left.[x]_{S(\alpha)} \cap X \neq \phi\right\}$ 
Definition 11: A subset $X$ of $U$ is said to be an $(R * S)(\alpha)$ - multigranular rough set if and only if

$R(\alpha) * S(\alpha) X \neq \overline{R(\alpha) * S(\alpha)} X$. Otherwise, we say $\mathrm{X}$ is $(R * S)(\alpha)$ - multigranular definable.

\section{RESULTS}

As noted above, for any two fuzzy proximity relations $\mathrm{R}$ and $\mathrm{S}$ defined over $\mathrm{U}$ and any $\alpha \in[0,1], R(\alpha)$ and $S(\alpha)$ are equivalence relations. So, the properties in Theorem 2 follow directly from the corresponding properties of optimistic multigranular rough sets established in [34]. Similarly, the properties in Theorem 3 follow from the corresponding properties of optimistic multigranular rough sets established in [35].

Theorem 2: We have

$$
\underline{R(\alpha)+S(\alpha)} X \subseteq X \subseteq \overline{R(\alpha)+S(\alpha)} X
$$

$$
\begin{aligned}
& R(\alpha)+S(\alpha) \phi=\overline{R(\alpha)+S(\alpha)} \phi=\phi \text { and } R(\alpha)+S(\alpha) U \\
& =\overline{R(\alpha)+S(\alpha)} U=U \\
& X \subseteq Y \Rightarrow R(\alpha)+S(\alpha) X \subseteq R(\alpha)+S(\alpha) Y \text { and } \\
& \overline{R(\alpha)+S(\alpha)} X \subseteq \overline{R(\alpha)+S(\alpha)} Y
\end{aligned}
$$

Theorem3: We have

$$
\begin{gathered}
\frac{R(\alpha) * S(\alpha)}{} X \subseteq X \subseteq \overline{R(\alpha) * S(\alpha)} X \\
\frac{R(\alpha) * S(\alpha) \phi}{R(\alpha) * S(\alpha)} U=\overline{R(\alpha) * S(\alpha)} \phi=\phi \text { and } \\
X \subseteq Y \Rightarrow \underline{R(\alpha) * S(\alpha)} U=U \\
\overline{R(\alpha) * S(\alpha)} X \subseteq S(\alpha) X \subseteq \underline{R(\alpha) * S(\alpha)} Y \text { and }
\end{gathered}
$$

The following results can be obtained from (22) to (25) where the inclusions can be strict.

Theorem 4: For any two fuzzy proximity relations $\mathrm{R}$ and $\mathrm{S}$ defined over $\mathrm{U}, X, Y \subseteq U$ and any $\alpha \in[0,1]$, we have

$$
\underline{R(\alpha)+S(\alpha)}(X \cup Y) \supseteq \underline{R(\alpha)+S(\alpha)}(X) \cup \underline{R(\alpha)+S(\alpha)}(Y)
$$

$$
\overline{R(\alpha)+S(\alpha)}(X \bigcup Y) \supseteq \overline{R(\alpha)+S(\alpha)}(X) \cup \overline{R(\alpha)+S(\alpha)}(Y)
$$

$\underline{R(\alpha)+S(\alpha)}(X \cap Y) \subseteq \underline{R(\alpha)+S(\alpha)}(X) \bigcap \underline{R(\alpha)+S(\alpha)}(Y)$

$$
\overline{R(\alpha)+S(\alpha)}(X \cap Y) \subseteq \overline{R(\alpha)+S(\alpha)}(X) \cap \overline{R(\alpha)+S(\alpha)}(Y)
$$

The following results can be obtained from (26) to (29) and Note. 3.

Theorem 5: For any two fuzzy proximity relations $\mathrm{R}$ and $\mathrm{S}$ defined over $\mathrm{U}, X, Y \subseteq U$ and any $\alpha \in[0,1]$, we have

$$
\underline{R(\alpha) * S(\alpha)}(X \bigcup Y) \supseteq \underline{R(\alpha) * S(\alpha)}(X) \cup \underline{R(\alpha) * S(\alpha)}(Y)
$$

$$
\overline{R(\alpha) * S(\alpha)}(X \bigcup Y)=\overline{R(\alpha) * S(\alpha)}(X) \bigcup \overline{R(\alpha) * S(\alpha)}(Y)
$$

$\underline{R(\alpha) * S(\alpha)}(X \cap Y)=\underline{R(\alpha) * S(\alpha)}(X) \cap \underline{R(\alpha) * S(\alpha)}(Y)$

$$
\overline{R(\alpha) * S(\alpha)}(X \cap Y) \subseteq \overline{R(\alpha) * S(\alpha)}(X) \cap \overline{R(\alpha) * S(\alpha)}(Y)
$$

Theorem 6: If $\alpha_{1} \geq \alpha_{2}$ and $\alpha_{1}, \alpha_{2} \in[0,1]$ then for any two fuzzy proximity relations $\mathrm{R}$ and $\mathrm{S}$ defined over $\mathrm{U}$ and any subset $\mathrm{X}$ of $\mathrm{U}$, we have

$$
\begin{aligned}
& \underline{R\left(\alpha_{2}\right)+S\left(\alpha_{2}\right) X \subseteq \underline{R\left(\alpha_{1}\right)+S\left(\alpha_{1}\right) X}} \\
& \overline{R\left(\alpha_{1}\right)+S\left(\alpha_{1}\right)} X \subseteq \overline{R\left(\alpha_{2}\right)+S\left(\alpha_{2}\right)} X \\
& \underline{R\left(\alpha_{2}\right) * S\left(\alpha_{2}\right) X \subseteq \underline{R\left(\alpha_{1}\right) * S\left(\alpha_{1}\right) X}} \\
& \overline{R\left(\alpha_{1}\right) * S\left(\alpha_{1}\right)} X \subseteq \overline{R\left(\alpha_{2}\right) * S\left(\alpha_{2}\right)} X
\end{aligned}
$$

Proof: Proof of (52):

$$
\begin{aligned}
& \text { Let } x \in \underline{R\left(\alpha_{2}\right)+S\left(\alpha_{2}\right) X} . \text { Then } \\
& {[x]_{R\left(\alpha_{2}\right)} \subseteq X \text { or }[x]_{\mathbb{S}_{\left(\alpha_{2}\right)}} \subseteq X \text {. Now, }} \\
& \quad y \in[x]_{R\left(\alpha_{1}\right)} \Rightarrow \mu_{R}(x, y) \geq \alpha_{1} \Rightarrow \mu_{R}(x, y) \geq \alpha_{2} \Rightarrow y \in[x]_{R\left(\alpha_{2}\right)}
\end{aligned}
$$

So, $[x]_{R_{\alpha_{1}}} \subseteq[x]_{R_{\alpha_{2}}}$. Similarly, $[x]_{S_{\alpha_{1}}} \subseteq[x]_{S_{\alpha_{2}}}$

Hence,

$$
\begin{gathered}
x \in \underline{R\left(\alpha_{2}\right)+S\left(\alpha_{2}\right) X} \Rightarrow[x]_{R\left(\alpha_{2}\right)} \subseteq X \text { or }[x]_{\mathrm{S}\left(\alpha_{2}\right)} \subseteq X \\
\Rightarrow[x]_{R\left(\alpha_{1}\right)} \subseteq X \text { or }[x]_{S\left(\alpha_{1}\right)} \subseteq X \Rightarrow x \in \underline{R\left(\alpha_{1}\right)+S\left(\alpha_{1}\right) X} .
\end{gathered}
$$

This completes the proof.

$$
\text { Proof of (53): }
$$

$$
x \in \overline{R\left(\alpha_{1}\right)+S\left(\alpha_{1}\right)} X . \Rightarrow[x]_{R\left(\alpha_{1}\right)} \cap X \neq \phi \text { and }[x]_{S\left(\alpha_{1}\right)} \cap X \neq \phi .
$$

Now using (52) it follows

that $[x]_{R\left(\alpha_{2}\right)} \cap X \neq \phi$ and $[x]_{\mathrm{S}\left(\alpha_{2}\right)} \cap X \neq \phi \quad$. Hence, 
$x \in \overline{R\left(\alpha_{2}\right)+S\left(\alpha_{2}\right)} X$. This completes the proof.

Proofs of (54) and (55) are similar. Only we have to replace 'or' by 'and'.

Next for any $\alpha \in[0,1]$, we denote the optimistic and pessimistic boundaries of a subset $\mathrm{X}$ of $\mathrm{U}$ with respect to two fuzzy proximity relations $\mathrm{R}$ and $\mathrm{S}$ over the fuzzy approximation space by $B N_{(R(\alpha)+S(\alpha))}(X)$ and $B N_{(R(\alpha) * S(\alpha))}(X)$ respectively and define as

$$
\begin{gathered}
B N_{(R(\alpha)+S(\alpha))} X=\overline{R(\alpha)+S(\alpha)} X \backslash \underline{R(\alpha)+S(\alpha) X} \\
\text { and } B N_{(R(\alpha) * S(\alpha))} X=\overline{R(\alpha) * S(\alpha)} X \backslash \underline{R(\alpha) * S(\alpha) X} X
\end{gathered}
$$

Corollary 1:If $\alpha_{1} \geq \alpha_{2}$ and $\alpha_{1}, \alpha_{2} \in[0,1]$ then for any two fuzzy proximity relations $\mathrm{R}$ and $\mathrm{S}$ defined over $\mathrm{U}$ and any subset $\mathrm{X}$ of $\mathrm{U}$, we have

$$
\begin{gathered}
B N_{\left(R\left(\alpha_{1}\right)+S\left(\alpha_{1}\right)\right)} X \subseteq B N_{\left(R\left(\alpha_{2}\right)+S\left(\alpha_{2}\right)\right)} X \\
B N_{\left(R\left(\alpha_{1}\right)+S\left(\alpha_{1}\right)\right)} X \subseteq B N_{\left(R\left(\alpha_{2}\right)+S\left(\alpha_{2}\right)\right)} X \\
\text { and } B N_{\left(R\left(\alpha_{1}\right) * S\left(\alpha_{1}\right)\right)} X \subseteq B N_{\left(R\left(\alpha_{2}\right) * S\left(\alpha_{2}\right)\right)} X .
\end{gathered}
$$

Proof: Directly follows from Theorem 4.5.

So, as a consequence we see that as $\alpha$ increases the uncertainty with respect to both the optimistic multigranulation and pessimistic multigranulation decreases. As mentioned in Note 2.1 above, for any two fuzzy proximity relations $\mathrm{R}$ and $\mathrm{S}, R \cup S$ and $R \cap S$ are fuzzy proximity relations. Hence, it is meaningful to talk about $(R \bigcup S)(\alpha),(R \bigcap S)(\alpha)$ and their multigranulations. We have the following result:

Theorem 7: For fuzzy proximity relations R, S, T, V on $\mathrm{U}$ and $\alpha$ be a chosen level value in $[0,1]$, then we have

$$
\begin{aligned}
& \frac{(R \cup S)(\alpha)+(T \bigcup V)(\alpha) X \subseteq}{(R(\alpha)+T(\alpha))} X \bigcup \underline{(S(\alpha)+V(\alpha))} X \\
& \overline{(R \bigcup S)(\alpha)+(T \bigcup V)(\alpha) X \supseteq} \\
& \overline{(R(\alpha)+T(\alpha))} X \bigcup \overline{(S(\alpha)+V(\alpha))} \\
& \overline{(R \cap S)(\alpha)+(T \cap V)(\alpha)} X \subseteq \\
& \overline{(R(\alpha)+T(\alpha))} X \cap \overline{(S(\alpha)+V(\alpha))} X
\end{aligned}
$$

Proof: Proof of (60):

$$
\begin{aligned}
& x \in(R \cup S)(\alpha)+(T \cup V)(\alpha) X \\
& \Leftrightarrow[x]_{(R \cup S)(\alpha)} \subseteq X \text { or }[x]_{(T \cup V)(\alpha)} \subseteq X \\
& \Rightarrow\left([x]_{R(\alpha)} \subseteq X \text { or }[x]_{S(\alpha)} \subseteq X\right) \text { or }\left([x]_{T(\alpha)} \subseteq X \text { or }[x]_{V(\alpha)} \subseteq X\right) \\
& \left.\Leftrightarrow\left([x]_{R(\alpha)} \subseteq X \text { or }[x]_{T(\alpha)} \subseteq X\right) \text { or }\left([x]_{S(\alpha)} \subseteq X \text { or }[x]_{V(\alpha)} \subseteq X\right)\right\} \\
& \Leftrightarrow x \in R(\alpha)+T(\alpha) X \\
& \Leftrightarrow x \in R(\alpha)+T(\alpha) X \bigcup S(\alpha)+V(\alpha) X
\end{aligned}
$$

This completes the proof. Proof of (61):

$$
\begin{gathered}
x \in \overline{(R(\alpha)+T(\alpha))} X \cup \overline{(S(\alpha)+V(\alpha))} X \\
\Leftrightarrow x \in \overline{(R(\alpha)+T(\alpha))} X \text { or } \overline{(S(\alpha)+V(\alpha))} X \\
\Leftrightarrow\left([x]_{R(\alpha)} \cap X \neq \phi \text { and }[x]_{T(\alpha)} \cap X \neq \phi\right) \text { or } \\
\left([x]_{S(\alpha)} \cap X \neq \phi \text { and }[x]_{V(\alpha)} \cap X \neq \phi\right) \\
\Leftrightarrow\left([x]_{R(\alpha)} \cap X \neq \phi \text { or }[x]_{S(\alpha)} \cap X \neq \phi\right) \text { and } \\
\left([x]_{T(\alpha)} \cap X \neq \phi \text { or }[x]_{V(\alpha)} \cap X \neq \phi\right) \text { and } \\
\left(\left([x]_{R(\alpha)} \cap X \neq \phi \text { or }[x]_{S(\alpha)} \cap X \neq \phi\right)\right. \text { and } \\
\left([x]_{T(\alpha)} \cap X \neq \phi \text { or }[x]_{V(\alpha)} \cap X \neq \phi\right) \\
\Rightarrow\left([x]_{R(\alpha)} \cap X \neq \phi \text { or }[x]_{S(\alpha)} \cap X \neq \phi\right) \text { and } \\
\left([x]_{T(\alpha)} \cap X \neq \phi \text { or }[x]_{V(\alpha)} \cap X \neq \phi\right) \\
\left.\Leftrightarrow\left(\left([x]_{R(\alpha)} \cap X\right) \cup\left([x]_{S(\alpha)} \cap X\right)\right) \neq \phi\right) \text { and } \\
\left.\left(\left([x]_{T(\alpha)} \cap X\right) \cup\left([x]_{V(\alpha)} \cap X\right)\right) \neq \phi\right) \\
\left.\Leftrightarrow\left(\left([x]_{R(\alpha)} \cup[x]_{S(\alpha)}\right) \cap X\right) \neq \phi\right) \text { and } \\
\left.\quad\left(\left([x]_{T(\alpha)} \cup[x]_{V(\alpha)}\right) \cap X\right) \neq \phi\right) \\
\left.\Leftrightarrow\left([x]_{(R \cup S)(\alpha)} \cap X\right) \neq \phi\right) \text { and } \\
\left.\quad\left([x]_{(T \cup V)(\alpha)} \cap X\right) \neq \phi\right) \\
\Leftrightarrow x \in \overline{(R \cup S)(\alpha)+(T \cup V)(\alpha) X}
\end{gathered}
$$

This completes the proof.

Proof of (62): We have

$$
\begin{gathered}
x \in \overline{(R \cap S)(\alpha)+(T \cap V)(\alpha)} X \\
\Leftrightarrow\left([x]_{(R \cap S)(\alpha)} \cap X \neq \phi\right) \text { and } \\
\left([x]_{(T \cap V)(\alpha)} \cap X \neq \phi\right) \\
\Rightarrow\left([x]_{R(\alpha)} \cap X \neq \phi \text { and }[x]_{S(\alpha)} \cap X \neq \phi\right) \text { and } \\
\left([x]_{T(\alpha)} \cap X \neq \phi \text { and }[x]_{V(\alpha)} \cap X \neq \phi\right) \\
\Rightarrow\left([x]_{R(\alpha)} \cap X \neq \phi \text { and }[x]_{T(\alpha)} \cap X \neq \phi\right) \text { and } \\
\left([x]_{S(\alpha)} \cap X \neq \phi \text { and }[x]_{V(\alpha)} \cap X \neq \phi\right) \\
\Leftrightarrow x \in \overline{R(\alpha)+T(\alpha)} X \text { and } \overline{S(\alpha)+V(\alpha)} X \\
\Leftrightarrow x \in \overline{R(\alpha)+T(\alpha)} X \cap \overline{S(\alpha)+V(\alpha)} X
\end{gathered}
$$

This completes the proof.

Note 7: We would like to note that no comparison can be made between the two expressions

$$
\begin{gathered}
(R \cap S)(\alpha)+(T \cap V)(\alpha) X \text { and } \\
(R(\alpha)+T(\alpha)) X \cap(S(\alpha)+V(\alpha)) X \\
\text { as is evident from the following: } \\
x \in R(\alpha)+T(\alpha) X \cap S(\alpha)+V(\alpha) X \\
\Leftrightarrow x \in R(\alpha)+T(\alpha) X \text { and } x \in S(\alpha)+V(\alpha) X
\end{gathered}
$$




$$
\begin{aligned}
\Leftrightarrow & \left([x]_{R(\alpha)} \subseteq X \text { or }[x]_{T(\alpha)} \subseteq X\right) \text { and } \\
& \left([x]_{S(\alpha)} \subseteq X \text { or }[x]_{V(\alpha)} \subseteq X\right) \\
\Leftarrow & \left([x]_{R(\alpha)} \subseteq X \text { and }[x]_{S(\alpha)} \subseteq X\right) \text { or } \\
& \left([x]_{T(\alpha)} \subseteq X \text { and }[x]_{V(\alpha)} \subseteq X\right) \ldots \ldots . . . \\
\Rightarrow & {[x]_{(R \cap S)(\alpha)} \subseteq X \text { or }[x]_{(T \cap V)(\alpha)} \subseteq X . } \\
\Leftrightarrow & x \in \underline{(R \cap S)(\alpha)+(T \cap V)(\alpha) X}
\end{aligned}
$$

From (a) and (b) it is clear that we cannot get a conclusion in any particular direction. However, we could provide an example where the one way inclusion holds and can be strict. All the inclusions from (60) to (62) can be strict. To establish this we provide some examples.

\section{Example 2:}

Let us take the following four fuzzy proximity relations $\mathrm{R}, \mathrm{S}, \mathrm{T}$ and $\mathrm{V}$ given in Tables $1,2,3$ and 4.Clearly these are fuzzy reflexive, fuzzy symmetric. Also, $\mu_{R}\left(x_{1}, x_{2}\right)=0.8, \mu_{R}\left(x_{2}, x_{6}\right)=0.3$ and

$\mu_{R}\left(x_{1}, x_{6}\right)=0.2$. So, fuzzy transitive property is not satisfied for R. Similarly,

$$
\mu_{S}\left(x_{1}, x_{2}\right)=0.8, \mu_{S}\left(x_{2}, x_{3}\right)=0.7 \text { and } \mu_{S}\left(x_{1}, x_{3}\right)=0.2 \text {. }
$$

Hence, $\mathrm{S}$ is also not fuzzy transitive. $\mathrm{T}$ and $\mathrm{V}$ can be shown to be fuzzy proximity relations similarly.

Table 1. Fuzzy Proximity Relation R

\begin{tabular}{|c|c|c|c|c|c|c|}
\hline $\mathrm{R}$ & $\mathrm{x} 1$ & $\mathrm{x} 2$ & $\mathrm{x} 3$ & $\mathrm{x} 4$ & $\mathrm{x} 5$ & $\mathrm{x} 6$ \\
\hline $\mathrm{x} 1$ & 1 & 0.8 & 0.6 & 0.3 & 0.5 & 0.2 \\
\hline $\mathrm{x} 2$ & 0.8 & 1 & 0.7 & 0.6 & 0.4 & 0.3 \\
\hline $\mathrm{x} 3$ & 0.6 & 0.7 & 1 & 0.9 & 0.6 & 0.5 \\
\hline $\mathrm{x} 4$ & 0.3 & 0.6 & 0.9 & 1 & 0.7 & 0.6 \\
\hline $\mathrm{x} 5$ & 0.5 & 0.4 & 0.6 & 0.7 & 1 & 0.9 \\
\hline $\mathrm{x} 6$ & 0.2 & 0.3 & 0.5 & 0.6 & 0.9 & 1 \\
\hline
\end{tabular}

Table 2. Fuzzy Proximity Relation S

\begin{tabular}{|c|c|c|c|c|c|c|}
\hline $\mathrm{S}$ & $\mathrm{x}_{1}$ & $\mathrm{x}_{2}$ & $\mathrm{x}_{3}$ & $\mathrm{x}_{4}$ & $\mathrm{x}_{5}$ & $\mathrm{x}_{6}$ \\
\hline $\mathrm{x}_{1}$ & 1 & 0.8 & 0.2 & 0.5 & 0.6 & 0.7 \\
\hline $\mathrm{x}_{2}$ & 0.8 & 1 & 0.4 & 0.7 & 0.5 & 0.2 \\
\hline $\mathrm{x}_{3}$ & 0.2 & 0.4 & 1 & 0.5 & 0.3 & 0.6 \\
\hline $\mathrm{x}_{4}$ & 0.5 & 0.7 & 0.5 & 1 & 0.9 & 0.7 \\
\hline $\mathrm{x}_{5}$ & 0.6 & 0.5 & 0.3 & 0.9 & 1 & 0.6 \\
\hline $\mathrm{x}_{6}$ & 0.7 & 0.2 & 0.6 & 0.7 & 0.6 & 1 \\
\hline
\end{tabular}

Table 3. Fuzzy Proximity Relation T

\begin{tabular}{|c|c|c|c|c|c|c|}
\hline $\mathrm{T}$ & $\mathrm{x}_{1}$ & $\mathrm{x}_{2}$ & $\mathrm{x}_{3}$ & $\mathrm{x}_{4}$ & $\mathrm{x}_{5}$ & $\mathrm{x}_{6}$ \\
\hline $\mathrm{x}_{1}$ & 1 & 1 & 0.9 & 0.9 & 0.5 & 0.5 \\
\hline $\mathrm{x}_{2}$ & 1 & 1 & 0.9 & 0.9 & 0.5 & 0.5 \\
\hline $\mathrm{x}_{3}$ & 0.9 & 0.9 & 1 & 1 & 0.8 & 0.8 \\
\hline $\mathrm{x}_{4}$ & 0.9 & 0.9 & 1 & 1 & 0.8 & 0.8 \\
\hline $\mathrm{x}_{5}$ & 0.5 & 0.5 & 0.8 & 0.8 & 1 & 1 \\
\hline $\mathrm{x}_{6}$ & 0.5 & 0.5 & 0.8 & 0.8 & 1 & 1 \\
\hline
\end{tabular}

Table 4. Fuzzy Proximity Relation V

\begin{tabular}{|c|c|c|c|c|c|c|}
\hline $\mathrm{V}$ & $\mathrm{x}_{1}$ & $\mathrm{x}_{2}$ & $\mathrm{x}_{3}$ & $\mathrm{x}_{4}$ & $\mathrm{x}_{5}$ & $\mathrm{x}_{6}$ \\
\hline $\mathrm{x}_{1}$ & 1 & 0.6 & 07 & 0.8 & 0.5 & 0.5 \\
\hline $\mathrm{x}_{2}$ & 0.6 & 1 & 0.4 & 0.7 & 0.8 & 0.3 \\
\hline $\mathrm{x}_{3}$ & 0.7 & 0.4 & 1 & 0.5 & 0.2 & 0.9 \\
\hline $\mathrm{x}_{4}$ & 0.8 & 0.7 & 0.5 & 1 & 0.6 & 0.7 \\
\hline $\mathrm{x}_{5}$ & 0.5 & 0.8 & 0.2 & 0.6 & 1 & 0.3 \\
\hline $\mathrm{x}_{6}$ & 0.5 & 0.3 & 0.9 & 0.7 & 0.3 & 1 \\
\hline
\end{tabular}

Table 5. Fuzzy Proximity Relation $R \cup S$

\begin{tabular}{|c|c|c|c|c|c|c|}
\hline$R \cup S$ & $\mathrm{x}_{1}$ & $\mathrm{x}_{2}$ & $\mathrm{x}_{3}$ & $\mathrm{x}_{4}$ & $\mathrm{x}_{5}$ & $\mathrm{x}_{6}$ \\
\hline $\mathrm{x}_{1}$ & 1 & 0.8 & 0.6 & 0.5 & 0.6 & 0.7 \\
\hline $\mathrm{x}_{2}$ & 0.8 & 1 & 0.7 & 0.7 & 0.5 & 0.3 \\
\hline $\mathrm{x}_{3}$ & 0.6 & 0.7 & 1 & 0.9 & 0.6 & 0.6 \\
\hline $\mathrm{x}_{4}$ & 0.5 & 0.7 & 0.9 & 1 & 0.9 & 0.7 \\
\hline $\mathrm{x}_{5}$ & 0.6 & 0.5 & 0.6 & 0.9 & 1 & 0.9 \\
\hline $\mathrm{x}_{6}$ & 0.7 & 0.3 & 0.6 & 0.7 & 0.9 & 1 \\
\hline
\end{tabular}

Table 6. Fuzzy Proximity Relation $R \cap S$

\begin{tabular}{|c|c|c|c|c|c|c|}
\hline$R \cap S$ & $\mathrm{x}_{1}$ & $\mathrm{x}_{2}$ & $\mathrm{x}_{3}$ & $\mathrm{x}_{4}$ & $\mathrm{x}_{5}$ & $\mathrm{x}_{6}$ \\
\hline $\mathrm{x}_{1}$ & 1 & 0.8 & 0.2 & 0.3 & 0.5 & 0.2 \\
\hline $\mathrm{x}_{2}$ & 0.8 & 1 & 0.4 & 0.6 & 0.4 & 0.2 \\
\hline $\mathrm{x}_{3}$ & 0.2 & 0.4 & 1 & 0.5 & 0.3 & 0.5 \\
\hline $\mathrm{x}_{4}$ & 0.3 & 0.6 & 0.5 & 1 & 0.7 & 0.6 \\
\hline $\mathrm{x}_{5}$ & 0.5 & 0.4 & 0.3 & 0.7 & 1 & 0.6 \\
\hline $\mathrm{x}_{6}$ & 0.2 & 0.2 & 0.5 & 0.6 & 0.6 & 1 \\
\hline
\end{tabular}

Table 7. Fuzzy Proximity Relation $T \cup V$

\begin{tabular}{|c|c|c|c|c|c|c|}
\hline$T \cup V$ & $\mathrm{x}_{1}$ & $\mathrm{x}_{2}$ & $\mathrm{x}_{3}$ & $\mathrm{x}_{4}$ & $\mathrm{x}_{5}$ & $\mathrm{x}_{6}$ \\
\hline $\mathrm{x}_{1}$ & 1 & 1 & 0.9 & 0.9 & 0.5 & 0.5 \\
\hline $\mathrm{x}_{2}$ & 1 & 1 & 0.9 & 0.9 & 0.8 & 0.5 \\
\hline $\mathrm{x}_{3}$ & 0.9 & 0.9 & 1 & 1 & 0.8 & 0.9 \\
\hline $\mathrm{x}_{4}$ & 0.9 & 0.9 & 1 & 1 & 0.8 & 0.8 \\
\hline $\mathrm{x}_{5}$ & 0.5 & 0.8 & 0.8 & 0.8 & 1 & 1 \\
\hline $\mathrm{x}_{6}$ & 0.5 & 0.5 & 0.9 & 0.8 & 1 & 1 \\
\hline
\end{tabular}

Table 8. Fuzzy Proximity Relation $T \cap V$

\begin{tabular}{|c|c|c|c|c|c|c|}
\hline$T \cap V$ & $\mathrm{x}_{1}$ & $\mathrm{x}_{2}$ & $\mathrm{x}_{3}$ & $\mathrm{x}_{4}$ & $\mathrm{x}_{5}$ & $\mathrm{x}_{6}$ \\
\hline $\mathrm{x}_{1}$ & 1 & 0.6 & 0.7 & 0.8 & 0.5 & 0.5 \\
\hline $\mathrm{x}_{2}$ & 0.6 & 1 & 0.4 & 0.7 & 0.5 & 0.3 \\
\hline $\mathrm{x}_{3}$ & 0.7 & 0.4 & 1 & 0.5 & 0.2 & 0.8 \\
\hline $\mathrm{x}_{4}$ & 0.8 & 0.7 & 0.5 & 1 & 0.6 & 0.7 \\
\hline $\mathrm{x}_{5}$ & 0.5 & 0.5 & 0.2 & 0.6 & 1 & 0.3 \\
\hline $\mathrm{x}_{6}$ & 0.5 & 0.3 & 0.8 & 0.7 & 0.3 & 1 \\
\hline
\end{tabular}

Here, let us take $\alpha=0.8$. Then

$$
\begin{gathered}
R_{\alpha}^{*}=\left\{\left\{x_{1}, x_{2}\right\},\left\{x_{3}, x_{4}\right\},\left\{x_{5}, x_{6}\right\}\right\}, \\
S_{\alpha}^{*}=\left\{\left\{x_{1}, x_{2}\right\},\left\{x_{3}\right\},\left\{x_{4}, x_{5}\right\},\left\{x_{6}\right\}\right\}, \\
T_{\alpha}^{*}=\left\{\left\{x_{1}, x_{2}, x_{3}, x_{4}, x_{5}, x_{6}\right\}\right\},
\end{gathered}
$$




$$
\begin{aligned}
& V_{\alpha}^{*}=\left\{\left\{x_{1}, x_{4}\right\},\left\{x_{2}, x_{5}\right\},\left\{x_{3}, x_{6}\right\}\right\} \text { and } \\
& (R \cup S)_{\alpha}^{*}=\left\{\left\{x_{1}, x_{2}\right\},\left\{x_{3}, x_{4}, x_{5}, x_{6}\right\}\right\}
\end{aligned}
$$

Let us take $X=\left\{x_{2}, x_{3}, x_{4}\right\}$.

Then $(R \bigcup S)(\alpha)+(T \bigcup V)(\alpha) X=\phi$,

$R(\alpha)+T(\alpha) X=\left\{x_{3}, x_{4}\right\}, S(\alpha)+V(\alpha) X=\left\{x_{3}\right\}$. So,

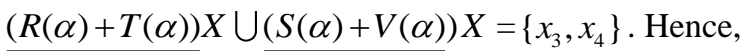
LHS $\subset$ RHS in (60).

$\overline{(R \bigcup S)(\alpha)+(T \bigcup V)(\alpha)} X=\left\{x_{1}, x_{2}, x_{3}, x_{4}, x_{5}, x_{6}\right\}$. $\overline{R(\alpha)+T(\alpha)} X=\left\{x_{1}, x_{2}, x_{3}, x_{4}\right\}$,

$\overline{S(\alpha)+V(\alpha)} X=\left\{x_{1}, x_{2}, x_{3}, x_{4}, x_{5}\right\}$. So, $(R(\alpha)+T(\alpha)) X \bigcup(S(\alpha)+V(\alpha)) X=\left\{x_{1}, x_{2}, x_{3}, x_{4}, x_{5}\right\}$.

Hence, LHS $\supset$ RHS in (61).

Continuing with this example we see that

$$
\begin{aligned}
& \frac{(R \cap S)(\alpha)+(T \cap V)(\alpha) X=\left\{x_{2}, x_{3}, x_{4}\right\},}{R(\alpha)+T(\alpha) X=\left\{x_{3}, x_{4}\right\}, S(\alpha)+V(\alpha) X=\left\{x_{3}\right\} .} \\
& \frac{R(\alpha)+T(\alpha) X}{L H S} \supset \underline{S(\alpha)+V(\alpha) X=\left\{x_{3}\right\} . \text { Hence, }} \\
& \text { RHS as claimed at (3). }
\end{aligned}
$$

We modify Example-2 to Example-3 below in order to provide an example for (62).

Example-3: Let us take $\mathrm{R}$ and $\mathrm{S}$ as same in Example-1 and also we choose $\alpha=0.8$. Let the fuzzy proximity relations $\mathrm{T}$ and $\mathrm{V}$ be given by tables 9 and 10 respectively.

Table 9. Fuzzy Proximity Relation T

\begin{tabular}{|c|c|c|c|c|c|c|}
\hline $\mathrm{T}$ & $\mathrm{x}_{1}$ & $\mathrm{x}_{2}$ & $\mathrm{x}_{3}$ & $\mathrm{x}_{4}$ & $\mathrm{x}_{5}$ & $\mathrm{x}_{6}$ \\
\hline $\mathrm{x}_{1}$ & 1 & 0.8 & 0.6 & 0.4 & 0.6 & 0.5 \\
\hline $\mathrm{x}_{2}$ & 0.8 & 1 & 0.6 & 0.3 & 0.6 & 0.5 \\
\hline $\mathrm{x}_{3}$ & 0.6 & 0.6 & 1 & 0.7 & 0.6 & 0.5 \\
\hline $\mathrm{x}_{4}$ & 0.4 & 0.3 & 0.7 & 1 & 0.5 & 0.6 \\
\hline $\mathrm{x}_{5}$ & 0.6 & 0.6 & 0.6 & 0.5 & 1 & 0.7 \\
\hline $\mathrm{x}_{6}$ & 0.5 & 0.5 & 0.5 & 0.6 & 0.7 & 1 \\
\hline
\end{tabular}

Table 10. Fuzzy Proximity Relation V

\begin{tabular}{|c|c|c|c|c|c|c|}
\hline $\mathrm{V}$ & $\mathrm{x}_{1}$ & $\mathrm{x}_{2}$ & $\mathrm{x}_{3}$ & $\mathrm{x}_{4}$ & $\mathrm{x}_{5}$ & $\mathrm{x}_{6}$ \\
\hline $\mathrm{x}_{1}$ & 1 & 0.6 & 0.7 & 0.8 & 0.5 & 0.5 \\
\hline $\mathrm{x}_{2}$ & 0.6 & 1 & 0.4 & 0.7 & 0.5 & 0.3 \\
\hline $\mathrm{x}_{3}$ & 0.7 & 0.4 & 1 & 0.5 & 0.2 & 0.8 \\
\hline $\mathrm{x}_{4}$ & 0.8 & 0.7 & 0.5 & 1 & 0.6 & 0.7 \\
\hline $\mathrm{x}_{5}$ & 0.5 & 0.5 & 0.2 & 0.6 & 1 & 0.3 \\
\hline $\mathrm{x}_{6}$ & 0.5 & 0.3 & 0.8 & 0.7 & 0.3 & 1 \\
\hline
\end{tabular}

Then the fuzzy proximity relations $T \cap V$ and $T \bigcup V$ are given by Tables 11 and 12 .
Table 11. Fuzzy Proximity Relation T

\begin{tabular}{|c|c|c|c|c|c|c|}
\hline$T \cap V$ & $\mathrm{x}_{1}$ & $\mathrm{x}_{2}$ & $\mathrm{x}_{3}$ & $\mathrm{x}_{4}$ & $\mathrm{x}_{5}$ & $\mathrm{x}_{6}$ \\
\hline $\mathrm{x}_{1}$ & 1 & 0.6 & 0.6 & 0.4 & 0.5 & 0.5 \\
\hline $\mathrm{x}_{2}$ & 0.6 & 1 & 0.4 & 0.3 & 0.6 & 0.3 \\
\hline $\mathrm{x}_{3}$ & 0.6 & 0.4 & 1 & 0.5 & 0.2 & 0.5 \\
\hline $\mathrm{x}_{4}$ & 0.4 & 0.3 & 0.5 & 1 & 0.5 & 0.6 \\
\hline $\mathrm{x}_{5}$ & 0.5 & 0.6 & 0.2 & 0.5 & 1 & 0.3 \\
\hline $\mathrm{x}_{6}$ & 0.5 & 0.3 & 0.5 & 0.6 & 0.3 & 1 \\
\hline
\end{tabular}

Table 12. Fuzzy Proximity Relation V

\begin{tabular}{|c|c|c|c|c|c|c|}
\hline$T \cap V$ & $\mathrm{x}_{1}$ & $\mathrm{x}_{2}$ & $\mathrm{x}_{3}$ & $\mathrm{x}_{4}$ & $\mathrm{x}_{5}$ & $\mathrm{x}_{6}$ \\
\hline $\mathrm{x}_{1}$ & 1 & 0.8 & 0.8 & 0.8 & 0.6 & 0.5 \\
\hline $\mathrm{x}_{2}$ & 0.8 & 1 & 0.6 & 0.9 & 0.7 & 0.5 \\
\hline $\mathrm{x}_{3}$ & 0.8 & 0.6 & 1 & 0.7 & 0.6 & 0.9 \\
\hline $\mathrm{x}_{4}$ & 0.8 & 0.9 & 0.7 & 1 & 0.6 & 0.7 \\
\hline $\mathrm{x}_{5}$ & 0.6 & 0.7 & 0.6 & 0.6 & 1 & 0.7 \\
\hline $\mathrm{x}_{6}$ & 0.5 & 0.5 & 0.9 & 0.7 & 0.7 & 1 \\
\hline
\end{tabular}

With this new set up,

$$
\begin{gathered}
T_{\alpha}^{*}=\left\{\left\{x_{1}, x_{2}\right\},\left\{x_{3}\right\},\left\{x_{4}\right\},\left\{x_{5}\right\},\left\{x_{6}\right\}\right\}, \\
V_{\alpha}^{*}=\left\{\left\{x_{1}, x_{2}, x_{3}, x_{4}, x_{6}\right\},\left\{x_{5}\right\}\right\} \text { and } \\
(T \cap V)_{\alpha}^{*}=\left\{\left\{x_{1}\right\},\left\{x_{2}\right\},\left\{x_{3}\right\},\left\{x_{4}\right\},\left\{x_{5}\right\},\left\{x_{6}\right\}\right\} . \\
(T \cup V)_{\alpha}^{*}=\left\{\left\{x_{1}, x_{2}, x_{3}, x_{4}, x_{6}\right\},\left\{x_{5}\right\}\right\} \text {. Let us take } \\
X=\left\{x_{1}, x_{3}, x_{6}\right\},
\end{gathered}
$$

Then $\overline{(R \cap S)(\alpha)+(T \cap V)(\alpha)} X=\left\{x_{1}, x_{3}, x_{6}\right\}$,

$$
\begin{aligned}
& \overline{R(\alpha)+T(\alpha)} X=\left\{x_{1}, x_{2}, x_{3}, x_{6}\right\} \text { and } \\
& \overline{S(\alpha)+V(\alpha)} X=\left\{x_{1}, x_{2}, x_{3}, x_{6}\right\} . \text { So, }
\end{aligned}
$$

$\overline{R(\alpha)+T(\alpha)} X \cap \overline{S(\alpha)+V(\alpha)} X=\left\{x_{1}, x_{2}, x_{3}, x_{6}\right\}$. So,

$$
\text { L.H.S } \subset \text { R.H.S in (60). }
$$

Theorem 8: Let $\mathrm{R}, \mathrm{S}, \mathrm{T}, \mathrm{V}$ be four fuzzy proximity relations on $\mathrm{U}$ and $\alpha$ be a chosen level value. Then

$$
\underline{(R * T)(\alpha)} X \bigcup \underline{(S * V)(\alpha)} X \supseteq \underline{(R \bigcup S)(\alpha) *(T \bigcup V)(\alpha) X}
$$

$$
\overline{(R \bigcup S)(\alpha) *(T \bigcup V)(\alpha)} X \supseteq \overline{(R * T)(\alpha)} X \bigcup \overline{(S * V)(\alpha)} X
$$

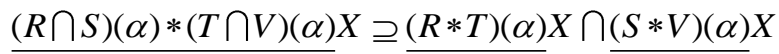

$$
\overline{(R \cap S)(\alpha) *(T \bigcap V)(\alpha)} X \subseteq \overline{(R * T)(\alpha)} X \cap \overline{(S * V)(\alpha)} X
$$

Proof: Proof of (63): We have

$$
\begin{aligned}
x \in & (R \cup S)(\alpha) *(T \cup V)(\alpha) X \\
\Leftrightarrow & {[x]_{(R \cup S)(\alpha)} \subseteq X \text { and }[x]_{(T \cup V)(\alpha)} \subseteq X } \\
\Rightarrow & \left([x]_{R(\alpha)} \subseteq X \text { and }[x]_{S(\alpha)} \subseteq X\right) \text { and } \\
& \left([x]_{T(\alpha)} \subseteq X \text { and }[x]_{V(\alpha)} \subseteq X\right)
\end{aligned}
$$




$$
\begin{aligned}
\Leftrightarrow & \left([x]_{R(\alpha)} \subseteq X \text { and }[x]_{T(\alpha)} \subseteq X\right) \text { and } \\
& \left([x]_{S(\alpha)} \subseteq X \text { and }[x]_{V(\alpha)} \subseteq X\right) \\
\Leftrightarrow & x \in \underline{R(\alpha) * T(\alpha) X \cap \underline{S(\alpha) * V(\alpha)} X} \\
\Rightarrow & x \in \underline{R(\alpha) * T(\alpha)} X \bigcup \underline{S(\alpha) * V(\alpha)} X
\end{aligned}
$$

This completes the proof.

Proof of (64): We have

$$
\begin{aligned}
x \in & \overline{(R * T)(\alpha)} X \cup \overline{(S * V)(\alpha)} X \\
\Leftrightarrow & x \in \overline{(R * T)(\alpha)} X \text { or } \mathrm{x} \in \overline{(S * V)(\alpha)} X \\
\Leftrightarrow & \left([x]_{R(\alpha)} \cap X \neq \phi \text { or }[x]_{T(\alpha)} \cap X \neq \phi\right) \text { or } \\
& \left([x]_{S(\alpha)} \cap X \neq \phi \text { or }[x]_{V(\alpha)} \cap X \neq \phi\right) \\
\Leftrightarrow & \left([x]_{R(\alpha)} \cap X \neq \phi \text { or }[x]_{S(\alpha)} \cap X \neq \phi\right) \text { or } \\
& \left([x]_{T(\alpha)} \cap X \neq \phi \text { or }[x]_{V(\alpha)} \cap X \neq \phi\right) \\
\Rightarrow & \left(\left([x]_{R(\alpha)} \cup[x]_{S(\alpha)}\right) \cap X \neq \phi\right) \text { or } \\
& \left(\left([x]_{T(\alpha)} \cup[x]_{V(\alpha)}\right) \cap X \neq \phi\right) \\
\Rightarrow & \left.\left([x]_{R(\alpha) \cup S(\alpha)} \cap X \neq \phi\right) \text { or }\left([x]_{T(\alpha) \cup V(\alpha)}\right) \cap X \neq \phi\right) \\
\Leftrightarrow & x \in \overline{(R \bigcup S)(\alpha) *(T \cup V)(\alpha) X}
\end{aligned}
$$

This completes the proof.

Proof of (65): We have

$$
\begin{aligned}
& x \in \underline{(R * T)_{\alpha}} X \cap(S * V)_{\alpha} X \\
& \Leftrightarrow x \in \underline{(R * T)_{\alpha}} X \text { and } \underline{(S * V)_{\alpha}} X \\
& \Leftrightarrow\left([x]_{R_{\alpha}} \subseteq X \text { and }[x]_{T_{\alpha}} \subseteq X\right) \text { and } \\
& \left([x]_{S_{\alpha}} \subseteq X \text { and }[x]_{V_{\alpha}} \subseteq X\right) \\
& \Leftrightarrow\left([x]_{R_{\alpha}} \subseteq X \text { and }[x]_{S_{\alpha}} \subseteq X\right) \text { and } \\
& \left([x]_{T_{\alpha}} \subseteq X \text { and }[x]_{V_{\alpha}} \subseteq X\right) \\
& \Rightarrow\left([x]_{R_{\alpha}} \cap[x]_{S_{\alpha}} \subseteq X\right) \text { and }\left([x]_{T_{\alpha}} \cap[x]_{V_{\alpha}} \subseteq X\right) \\
& \Rightarrow\left([x]_{(R \cap S)_{\alpha}} \subseteq X\right) \text { and }\left([x]_{(T \cap V)_{\alpha}} \subseteq X(\mathrm{By}(13))\right. \\
& \Leftrightarrow x \in(R \cap S)_{\alpha} *(T \cap V)_{\alpha} X
\end{aligned}
$$

This completes the proof.

Proof of (66): We have

$$
\begin{aligned}
x \in & \overline{(R \cap S)_{\alpha} *(T \cap V)_{\alpha}} X \\
\Leftrightarrow & \left([x]_{(R \cap S)_{\alpha}} \cap X \neq \phi\right) \text { or }\left([x]_{(T \cap V)_{\alpha}} \cap X \neq \phi\right) \\
\Rightarrow & \left.\left(\left([x]_{R(\alpha)} \cap X \neq \phi\right) \text { and }\left([x]_{S(\alpha)}\right) \cap X \neq \phi\right)\right) \text { or } \\
& \left.\left(\left([x]_{T(\alpha)} \cap X \neq \phi\right) \text { and }\left([x]_{V(\alpha)}\right) \cap X \neq \phi\right)\right) \\
\Rightarrow & \left(\left([x]_{R(\alpha)} \cap X \neq \phi\right) \text { or }\left([x]_{T(\alpha)} \cap X \neq \phi\right)\right. \text { and } \\
& \left.\left.\left(\left([x]_{S(\alpha)}\right) \cap X \neq \phi\right) \text { or }\left([x]_{V(\alpha)}\right) \cap X \neq \phi\right)\right) \\
\Leftrightarrow & x \in \overline{(R(\alpha) * T(\alpha)} X \text { and } x \in \overline{S(\alpha) * V(\alpha)} X \\
\Leftrightarrow & x \in \overline{R(\alpha) * T(\alpha)} X \cap \overline{S(\alpha) * V(\alpha)} X
\end{aligned}
$$

This completes the proof.

Note 8: All the inclusions from (63) to (66) can be strict.
(63): Let us follow example-1 above and $X=\left\{x_{1}, x_{3}, x_{6}\right\}$. Then

$$
\begin{aligned}
& \frac{(R * T)(\alpha)}{(R \cup S)(\alpha)} X=\phi, \quad \underline{(S * V)(\alpha) X} X=\left\{x_{3}, x_{6}\right\} . \text { So, } \\
& \underline{(T \cup V)(\alpha) X}=\left\{x_{3}, x_{6}\right\} . \text { Also, }
\end{aligned}
$$

$(R \bigcup S)(\alpha) *(T \bigcup V)(\alpha) X=\phi$. So, LHS $\supset$ RHS in (63).

(64): Let us follow example- 2 above and take $\alpha=0.9$. Then

$$
\begin{gathered}
R_{\alpha}^{*}=\left\{\left\{x_{1}\right\},\left\{x_{2}\right\},\left\{x_{3}, x_{4}\right\},\left\{x_{5}, x_{6}\right\}\right\}, \\
S_{\alpha}^{*}=\left\{\left\{x_{1}\right\},\left\{x_{2}\right\},\left\{x_{3}\right\},\left\{x_{4}, x_{5}\right\},\left\{x_{6}\right\}\right\}, \\
T_{\alpha}^{*}=\left\{\left\{x_{1}\right\},\left\{x_{2}\right\},\left\{x_{3}\right\},\left\{x_{4}\right\},\left\{x_{5}\right\},\left\{x_{6}\right\}\right\} \\
\text { and } V_{\alpha}^{*}=\left\{\left\{x_{1}\right\},\left\{x_{2}, x_{4}\right\},\left\{x_{3}, x_{6}\right\},\left\{x_{5}\right\}\right\} .
\end{gathered}
$$

Also, $(T \cup V)_{\alpha}^{*}=\left\{\left\{x_{1}\right\},\left\{x_{2}, x_{4}\right\},\left\{x_{3}, x_{6}\right\},\left\{x_{5}\right\}\right\}$ and

$$
(R \cup S)_{\alpha}^{*}=\left\{\left\{x_{1}\right\},\left\{x_{2}\right\},\left\{x_{3}, x_{4}, x_{5}, x_{6}\right\}\right\} .
$$

We take $X=\left\{x_{1}, x_{2}, x_{3}\right\}$. So,

$$
\begin{gathered}
\overline{(R \bigcup S)(\alpha) *(U \bigcup V)(\alpha)} X=\left\{x_{1}, x_{2}, x_{3}, x_{4}, x_{5}, x_{6}\right\}, \\
\overline{(R * T)(\alpha)} X=\left\{x_{1}, x_{2}, x_{3}, x_{4}\right\} \text { and } \\
\overline{(S * V)(\alpha)} X=\left\{x_{1}, x_{2}, x_{3}, x_{4}, x_{6}\right\} .
\end{gathered}
$$

Hence, $\overline{(R * T)(\alpha)} X \bigcup \overline{(S * V)(\alpha)} X=\left\{x_{1}, x_{2}, x_{3}, x_{4}, x_{6}\right\}$. This shows that LHS $\supset$ RHS in (64).

(65): Let $X=\left\{x_{1}, x_{3}, x_{6}\right\}$.

Then $(R \cap S)(\alpha) *(T \cap V)(\alpha) X=\left\{x_{3}, x_{6}\right\}$.

Also, $(R * T)(\alpha) X \cap(S * V)(\alpha) X=\phi$. Hence,

LHS $\supset$ RHS in (65).

(66):We use example-2 here. Let $X=\left\{x_{1}, x_{2}, x_{6}\right\}$. Then

$$
\begin{gathered}
\overline{(R \cap S)(\alpha) *(T \cap V)(\alpha)} X=\left\{x_{1}, x_{2}, x_{6}\right\}, \\
\overline{(R * T)(\alpha)} X=\left\{x_{1}, x_{2}, x_{3}, x_{4}, x_{5}, x_{6}\right\} \text { and } \\
\overline{(S * V)(\alpha)} X=\left\{x_{1}, x_{2}, x_{5}, x_{6}\right\} . \text { So, } \\
\overline{(R * T)(\alpha)} X \cap \overline{(S * V)(\alpha)} X=\left\{x_{1}, x_{2}, x_{5}, x_{6}\right\} . \text { Hence } \\
L H S \subset R H S \text { holds true in }(66) .
\end{gathered}
$$

\section{AN EXAMPLE FOR REAL LIFE APPLICATION}

We know that Rough Set theory has many applications in Decision Science. Here, we give one such example of Predicting whether it will rain or not.

Let us take an information system "Rain Prediction" with the conditional attributes: Humidity $(\mathrm{H})$, Climate Zone (C) and Temperature (T) and Decision Attribute Rain (R).

The attribute domains are as follows:

$\mathrm{A}=\{$ High, Low, Medium $\}, \mathrm{C}=\{$ Temperate, Tropical, 
Frigid $\}, T=\{$ High, Low, Medium $\}$, Rain $=\{$ Yes, No $\}$

Following is the table giving information for 6 different places $\mathrm{P}_{1}, \mathrm{P}_{2}, \mathrm{P}_{3}, \mathrm{P}_{4}, \mathrm{P}_{5}$ and $\mathrm{P}_{6}$.

Table 13. Climate Information Table

\begin{tabular}{|c|c|c|c|c|}
\hline Place & Humidity & Climate Zone & Temperature & Rain \\
\hline P1 & High & Tropical & High & Yes \\
\hline P2 & Medium & Temperate & High & No \\
\hline P3 & Medium & Temperate & Medium & Yes \\
\hline P4 & Low & Temperate & Medium & No \\
\hline P5 & Medium & Tropical & Low & Yes \\
\hline P6 & Low & Frigid & Low & No \\
\hline
\end{tabular}

Here, $\mathrm{U}=\left\{\mathrm{P}_{1}, \mathrm{P}_{2}, \mathrm{P}_{3}, \mathrm{P}_{4}, \mathrm{P}_{5}, \mathrm{P}_{6}\right\}$. Following are the fuzzy proximity relations $\mathrm{H}$ (for humidity), $\mathrm{C}$ (for climate zone), $\mathrm{T}$ (for Temperature) respectively:

Table 14. Fuzzy Proximity Relation for Humidity

\begin{tabular}{|c|c|c|c|}
\hline $\mathrm{H}$ & High & Medium & Low \\
\hline High & 1 & 0.8 & 0.5 \\
\hline Medium & 0.8 & 1 & 0.7 \\
\hline Low & 0.5 & 0.7 & 1 \\
\hline
\end{tabular}

Table 15.Fuzzy Proximity Relation for Climate Zone

\begin{tabular}{|c|c|c|c|}
\hline Climate Zone & Temperate & Tropical & Frigid \\
\hline Temperate & 1 & 0.7 & 0.2 \\
\hline Tropical & 0.7 & 1 & 0.4 \\
\hline Frigid & 0.2 & 0.4 & 1 \\
\hline
\end{tabular}

Table 16. Fuzzy Proximity Relation for Temperature

\begin{tabular}{|c|c|c|c|}
\hline Temperature & High & Medium & Low \\
\hline High & 1 & 0.9 & 0.5 \\
\hline Medium & 0.9 & 1 & 0.8 \\
\hline Low & 0.5 & 0.8 & 1 \\
\hline
\end{tabular}

Thus, fuzzy proximity relation for humidity on $\mathrm{U}$ derived from Table 13 and Table 14 is:

Table 17.Table for Fuzzy Proximity Relation Humidity on U

\begin{tabular}{|c|c|c|c|c|c|c|}
\hline Humidity & $\mathrm{P}_{1}$ & $\mathrm{P}_{2}$ & $\mathrm{P}_{3}$ & $\mathrm{P}_{4}$ & $\mathrm{P}_{5}$ & $\mathrm{P}_{6}$ \\
\hline $\mathrm{P}_{1}$ & 1 & 0.8 & 0.8 & 0.5 & 0.8 & 0.5 \\
\hline $\mathrm{P}_{2}$ & 0.8 & 1 & 1 & 0.7 & 1 & 0.7 \\
\hline $\mathrm{P}_{3}$ & 0.8 & 1 & 1 & 0.7 & 1 & 0.7 \\
\hline $\mathrm{P}_{4}$ & 0.5 & 0.7 & 0.7 & 1 & 0.7 & 1 \\
\hline $\mathrm{P}_{5}$ & 0.8 & 1 & 1 & 0.7 & 1 & 0.7 \\
\hline $\mathrm{P}_{6}$ & 0.5 & 0.7 & 0.7 & 1 & 0.7 & 1 \\
\hline
\end{tabular}

For the domain attribute Climate Zone, the fuzzy proximity relation derived from Table 13 and Table 15 is:
Table 18. Table for Fuzzy Proximity Relation Climate Zone on U

\begin{tabular}{|c|c|c|c|c|c|c|}
\hline Climate Zone & $\mathrm{P}_{1}$ & $\mathrm{P}_{2}$ & $\mathrm{P}_{3}$ & $\mathrm{P}_{4}$ & $\mathrm{P}_{5}$ & $\mathrm{P}_{6}$ \\
\hline $\mathrm{P}_{1}$ & 1 & 0.7 & 0.7 & 0.7 & 1 & 0.4 \\
\hline $\mathrm{P}_{2}$ & 0.7 & 1 & 1 & 1 & 0.7 & 0.2 \\
\hline $\mathrm{P}_{3}$ & 0.7 & 1 & 1 & 1 & 0.7 & 0.2 \\
\hline $\mathrm{P}_{4}$ & 0.7 & 1 & 1 & 1 & 0.7 & 0.2 \\
\hline $\mathrm{P}_{5}$ & 1 & 0.7 & 0.7 & 0.7 & 1 & 0.4 \\
\hline $\mathrm{P}_{6}$ & 0.4 & 0.2 & 0.2 & 0.2 & 0.4 & 1 \\
\hline
\end{tabular}

Also, for the domain attribute Temperature, the fuzzy proximity relation derived from Table 13 and Table 16 is shown in Table 19.

Table 19. Table for Fuzzy Proximity Relation Temperature on U

\begin{tabular}{|c|c|c|c|c|c|c|}
\hline Temperature & $\mathrm{P}_{1}$ & $\mathrm{P}_{2}$ & $\mathrm{P}_{3}$ & $\mathrm{P}_{4}$ & $\mathrm{P}_{5}$ & $\mathrm{P}_{6}$ \\
\hline $\mathrm{P}_{1}$ & 1 & 1 & 0.9 & 0.9 & 0.5 & 0.5 \\
\hline $\mathrm{P}_{2}$ & 1 & 1 & 0.9 & 0.9 & 0.5 & 0.5 \\
\hline $\mathrm{P}_{3}$ & 0.9 & 0.9 & 1 & 1 & 0.8 & 0.8 \\
\hline $\mathrm{P}_{4}$ & 0.9 & 0.9 & 1 & 1 & 0.8 & 0.8 \\
\hline $\mathrm{P}_{5}$ & 0.5 & 0.5 & 0.8 & 0.8 & 1 & 1 \\
\hline $\mathrm{P}_{6}$ & 0.5 & 0.5 & 0.8 & 0.8 & 1 & 1 \\
\hline
\end{tabular}

Now, let us take the target set $X=\left\{P_{1}, P_{3}, P_{5}\right\}$ corresponding to the "Yes" category of the decision attribute "Rain" and find out which places are similar with respect to the two granularities of Humidity and Climate Zone.

Let us take $\alpha$ as 0.8 .The partitions of $\mathrm{U}$ induced from the above fuzzy proximity relations Humidity and Climate as provided in Tables 16 and 17are:

$$
\begin{aligned}
H_{\alpha}^{*} & =\left\{\left\{P_{1}, P_{2}, P_{3}, P_{5}\right\},\left\{P_{4}, P_{6}\right\}\right\} \text { and } \\
C_{\alpha}^{*} & =\left\{\left\{P_{1}, P_{5}\right\},\left\{P_{2}, P_{3}, P_{4}\right\},\left\{P_{6}\right\}\right\} .
\end{aligned}
$$

The optimistic multigranular lower and upper approximations of $\mathrm{X}$ with respect to Humidity and Climate are as follows:

$$
\begin{aligned}
\underline{H(\alpha)+C(\alpha)} X & =\left\{P_{1}, P_{5}\right\} \text { and } \\
\overline{H(\alpha)+C(\alpha)} X & =\left\{P_{1}, P_{2}, P_{3}, P_{5}\right\}
\end{aligned}
$$

Similarly, the pessimistic multigranular lower and upper approximations of $\mathrm{X}$ with respect to Humidity and Climate are as follows:

$$
\begin{gathered}
\frac{H(\alpha) * C(\alpha) X}{}=\{\} \text { and } \\
\overline{H(\alpha) * C(\alpha)} X=\left\{P_{1}, P_{2}, P_{3}, P_{4}, P_{5}\right\}
\end{gathered}
$$


Hence, we can say from (67) that the set of places with similar humidity or in the same climate zone to a degree of 0.8 with rain prediction "yes" are $P_{1}$ and $P_{5}$. Also, the set of places with at least one place with same humidity and at least one place in the same climate zone to a degree 0.8 with rain prediction "yes" are $P_{1}, P_{2}, P_{3}$ and $P_{5}$. So that, we can positively or unambiguously classify $\mathrm{P}_{1}$ and $\mathrm{P}_{5}$ places as belonging to "Yes" category of "Rain" with respect to similar humidity or in the same climate zone to a degree of 0.8 . Also, we cannot unambiguously classify $\mathrm{P}_{1}, \mathrm{P}_{2}, \mathrm{P}_{3}$ and $\mathrm{P}_{5}$ as belonging to "No" category of "Rain" with respect to similar humidity and in the same climate zone to a degree of 0.8 .

Again, from (68) we conclude that we don't have any place which have similar humidity and are in the same climate zone to a degree of 0.8 with rain prediction "yes". And the set of places with at least one place with same humidity or at least one place in the same climate zone to a degree 0.8 with rain prediction "yes" are $P_{1}, P_{2}, P_{3}, P_{4}$ and $P_{5}$. So, we cannot classify any place as belonging to the "yes" category of "Rain" with respect to similar humidity similar humidity and in the same climate zone to a degree of 0.8 . Also, we cannot unambiguously classify all places $P_{1}, P_{2}, P_{3}, P_{4}$ and $P_{5}$ as belonging to the "No" category of "Rain" with respect to similar humidity and in the same climate zone to a degree of 0.8 .

Here, if we take the union of the fuzzy proximity relations $\mathrm{H}$ and $\mathrm{C}$, we get the relation $(H \cup C)$ as shown in Table 20.

Table 20. Fuzzy Proximity Relation for $(H \cup C)$

\begin{tabular}{|c|c|c|c|c|c|c|}
\hline Place & $\mathrm{P}_{1}$ & $\mathrm{P}_{2}$ & $\mathrm{P}_{3}$ & $\mathrm{P}_{4}$ & $\mathrm{P}_{5}$ & $\mathrm{P}_{6}$ \\
\hline $\mathrm{P}_{1}$ & 1 & 0.8 & 0.8 & 0.7 & 1 & 0.5 \\
\hline $\mathrm{P}_{2}$ & 0.8 & 1 & 1 & 1 & 1 & 0.7 \\
\hline $\mathrm{P}_{3}$ & 0.8 & 1 & 1 & 1 & 1 & 0.7 \\
\hline $\mathrm{P}_{4}$ & 0.7 & 1 & 1 & 1 & 0.7 & 1 \\
\hline $\mathrm{P}_{5}$ & 1 & 1 & 1 & 0.7 & 1 & 0.7 \\
\hline $\mathrm{P}_{6}$ & 0.5 & 0.7 & 0.7 & 1 & 0.7 & 1 \\
\hline
\end{tabular}

Hence, $(H \cup C)_{\alpha}^{*}=\left\{P_{1}, P_{2}, P_{3}, P_{4}, P_{5}, P_{6}\right\}$. So,

$$
\begin{gathered}
\frac{(H \cup C)(\alpha) X}{}=\{\} \text { and } \\
(H \cup C)(\alpha) X=\left\{P_{1}, P_{2}, P_{3}, P_{4}, P_{5}, P_{6}\right\}
\end{gathered}
$$

From (69) we observe that if we take Humidity or Climate zone (may be both) and a similarity to degree 0.8then we cannot unambiguously classify any of the places as belonging to the "Yes" category of "Rain" and similarly we cannot unambiguously classify any of the places as belonging to the "No" category of "Rain".

But taking Humidity and Climate Zone as two separate independent granularities simultaneously under multigranulation for predicting whether it will Rain or not, is much more useful than taking the Union of the two Relations and using it as a single granularity for the prediction.

Now, consider another value of $\alpha$ as 0.7 .The partitions $H_{\alpha}^{*}$ and $C_{\alpha}^{*}$ of $\mathrm{U}$ induced from the above tables with respect to Humidity and Climate respectively are given by

$$
\begin{gathered}
H_{\alpha}^{*}=\left\{\mathrm{P}_{1}, \mathrm{P}_{2}, \mathrm{P}_{3}, \mathrm{P}_{4}, \mathrm{P}_{5}, \mathrm{P}_{6}\right\} \text { and } \\
C_{\alpha}^{*}=\left\{\mathrm{P}_{1}, \mathrm{P}_{2}, \mathrm{P}_{3}, \mathrm{P}_{4}, \mathrm{P}_{5}, \mathrm{P}_{6}\right\} . \text { So, } \\
\frac{H(\alpha)+C(\alpha) X=\{\} \text { and }}{H(\alpha)+C(\alpha) X=\left\{P_{1}, P_{2}, P_{3}, P_{4}, P_{5}, P_{6}\right\}}
\end{gathered}
$$

Also, we have

$$
\begin{gathered}
\frac{H(\alpha) * C(\alpha) X}{}=\{\} \text { and } \\
\overline{H(\alpha) * C(\alpha)} X=\left\{P_{1}, P_{2}, P_{3}, P_{4}, P_{5}, P_{6}\right\}
\end{gathered}
$$

So, from (70) and (71) we see that there is no distinction between the two types of multigranulations.

Finally, taking $\alpha=0.9$, we get the partitions of $\mathrm{U}$ induced from the above tables with respect to Humidity and Climate as

$$
\begin{aligned}
& H_{\alpha}^{*}=\left\{\left\{P_{1}\right\},\left\{P_{2}, P_{3}, P_{5}\right\},\left\{P_{4}, P_{6}\right\}\right\} \text { and } \\
& C_{\alpha}^{*}=\left\{\left\{\mathrm{P}_{1}, \mathrm{P}_{5}\right\},\left\{\mathrm{P}_{2}, \mathrm{P}_{3}, \mathrm{P}_{4}, \mathrm{P}_{6}\right\}\right\} \text {. So, } \\
& H(\alpha)+C(\alpha) X=\left\{P_{1}, P_{5}\right\} \text { and } \\
& \overline{H(\alpha)+C(\alpha)} X=\left\{P_{1}, P_{2}, P_{3}, P_{4}, P_{5}, P_{6}\right\} \\
& H(\alpha) * C(\alpha) X=\left\{P_{1}\right\} \text { and } \\
& \overline{H(\alpha) * C(\alpha)} X=\left\{P_{1}, P_{2}, P_{3}, P_{4}, P_{5}, P_{6}\right\}
\end{aligned}
$$

Let us put all the lower and upper approximations of $\mathrm{X}$ for different degrees of similarities obtained above for the purpose of comparison.

Table 21. Table for Fuzzy Proximity Relation Climate Zone on U

\begin{tabular}{|c|c|c|c|c|}
\hline$\alpha$ & $H(\alpha)+C(\alpha) X$ & $\overline{H(\alpha)+C(\alpha)} X$ & $\underline{H(\alpha) * C(\alpha) X}$ & $\overline{H(\alpha) * C(\alpha)} X$ \\
\hline 0.7 & \{\} & $\left\{P_{1}, P_{2}, P_{3}, P_{4}, P_{5}, P_{6}\right\}$ & \{\} & $\left\{P_{1}, P_{2}, P_{3}, P_{4}, P_{5}, P_{6}\right\}$ \\
\hline 0.8 & $\left\{P_{1}, P_{5}\right\}$ & $\left\{P_{1}, P_{2}, P_{3}, P_{5}\right\}$ & \{\} & $\left\{P_{1}, P_{2}, P_{3}, P_{4}, P_{5}, P_{6}\right\}$ \\
\hline 0.9 & $\left\{P_{1}, P_{5}\right\}$ & $\left\{P_{1}, P_{2}, P_{3}, P_{5}\right\}$ & $\left\{P_{1}\right\}$ & $\left\{P_{1}, P_{2}, P_{3}, P_{4}, P_{5}, P_{6}\right\}$ \\
\hline
\end{tabular}


It is observed that as the value of $\alpha$ increases, our ability to classify data instances unambiguously increases too. This makes sense because $\alpha$ denotes the level of fuzziness that is tolerated, which is compatible with our theoretical observation in Theorem 6 earlier. Also, the optimistic and pessimistic multigranular boundary regions of $\mathrm{X}$ with respect to Humidity and Climate are as follows.

Table 22. Table for Boundaries of X for Different Values of $\alpha$

\begin{tabular}{|c|c|c|}
\hline$\alpha$ & $B N_{H(\alpha)+C(\alpha)} X$ & $B N_{H(\alpha) * C(\alpha)} X$ \\
\hline 0.7 & $\left\{P_{1}, P_{2}, P_{3}, P_{4}, P_{5}, P_{6}\right\}$ & $\left\{P_{1}, P_{2}, P_{3}, P_{4}, P_{5}, P_{6}\right\}$ \\
\hline 0.8 & $\left\{P_{2}, P_{3}\right\}$ & $\left\{P_{1}, P_{2}, P_{3}, P_{4}, P_{5}, P_{6}\right\}$ \\
\hline 0.9 & $\left\{P_{2}, P_{3}\right\}$ & $\left\{P_{2}, P_{3}, P_{4}, P_{5}, P_{6}\right\}$ \\
\hline
\end{tabular}

It can be seen that the boundary region decreases with the increasing value of $\alpha$. This means that the roughness or uncertainty decreases with the increasing value of $\alpha$. This is compatible with our result in Corollary 1.

\section{CONCLUSIONS}

In this paper, we introduced the notions of optimistic and pessimistic multigranular rough sets on fuzzy approximation spaces, which are the multigranular versions of the rough sets on fuzzy approximation spaces. These notions are more general than the basic optimistic and pessimistic multigranular rough sets as the basic requirement of equivalence relations have been liberalized. Several properties of these notions involving lower approximation, upper approximation of union and intersection of multigranulation have been established. Through a real life application we illustrated the utility of these new models.

\section{REFERENCES}

[1] A. Skowron and J. Stepaniuk, "Tolerance Approximation Spaces", Fundamenta Informaticae, vol. 27 (2-3), 245 253, 1996.

[2] B.K.Tripathy, "Rough Sets on Intuitionistic Fuzzy Approximation Spaces", IEEE Intelligent Systems, 4155526, 2006, pp.776-779..

[3] B.K. Tripathy, "Rough Sets on Fuzzy Approximation Spaces and Intuitionistic Fuzzy Approximation Spaces". In Studies in Computational Intelligence, Rough Set Theory: A True Landmark in Data Analysis, Vol. 174, Ajith Abraham, Falcon, Rafael Bello, Eds., Springer Verlag, 2009, pp. 03-44. doi:10.1007/978-3-540-89921$1 \_1$

[4] B.K. Tripathy and M. Nagaraju, "On Some Topological Properties of Pessimistic Multigranular Rough Sets", International Journal of Intelligent Systems and Applications, vol. 4(8), pp. 10-17, 2012.

[5] B.K.Tripathy and M. Nagaraju: Study of Covering Based Multi Granular Rough Sets and their Topological Properties, I.J. Information Technology and Computer Science, 08, pp. 61-67, 2015.

[6] B.K.Tripathy and M. Nagaraju, "Topological Properties of Incomplete Multigranulation based on Rough Fuzzy Sets", 2012 Communications in Computer and
Information Science 270 CCIS (PART II), 2012, pp.92102.

[7] B.K.Tripathy, G. K. Panda and A. Mitra, "Some Concepts of Incomplete Multigranulation based on Rough Intuitionistic Fuzzy Sets", Advances in Intelligent and Soft Computing, 166 AISC (Vol.1), 2012, pp.683-693.

[8] B.K. Tripathy and R. Raghavan, "Some Algebraic properties of Multigranulations and an Analysis of Multigranular Approximations of Classifications", International Journal of Information Technology and Computer Science, vol. 5(7), pp. 63-70, 2013.

[9] B.K. Tripathy and D.P. Acharjya, "Rough Set on Fuzzy Approximation Space and Applications to Distributed knowledge Systems", International Journal of Artificial intelligence and Soft Computing, vol. 1(1), pp. 1-15, 2008.

[10] B.K. Tripathy, S.S. Gantayat and D. Mohanty, "Properties of Rough Sets on Fuzzy Approximation Spaces and Knowledge Representation", In P. Dutta et al. (Eds).Proceeding of the National Conference on Recent Trends in Intelligent Computing, Kalyani Govt. Engineering College (17-19 November, 2006), 2006, pp. 3-8.

[11] B.K.Tripathy and A. Mitra, "On the Approximate Equalities of Multigranular Rough Sets and Approximate Reasoning, $20134^{\text {th }}$ International Conference on Computing, Communication and Network Technologies, ICCCNT 20136726771.

[12] B.K. Tripathy, "Multigranular Computing through Rough Sets", In B.K.Tripathy and D.P.Acharjya (Eds.), Advances in Secure Computing, Internet Services and Applications, AISPE Series, IGI Publications, 2014, pp.134.

[13] B.K.Tripathy and K. Govindarajulu, "On Covering Based Pessimistic Multigranular Rough Sets, Proceedings-2014 $6^{\text {th }}$ International Conference on Computational Intelligence and Communication Networks, CICN 2014 7065575, 2014, pp.708-713.

[14] B.K.Tripathy and A. Mitra, "On Algebraic and Topological Properties of Neighbourhood Based Multigranular Rough Sets, 2014 International Conference on Computer Communication and Informatics: Ushering in Technologies of Tomorrow an, Today, ICCCI 2014 6921770

[15] B.K.Tripathy, P. Saraf and S. C. Parida, "On Multigranular Approximate Rough Equivalence of sets and Approximate Reasoning, Smart Innovation, Systems and Technologies, 32, 2015, pp.605-616.

[16] B.K.Tripathy and M. Nagaraju: Topological Properties of Incomplete Multigranulation Based on Fuzzy Rough Sets, Obcom 2011 conference, VIT, Vellore from 9-11 Dec, 2011.

[17] B.K. Tripathy and S.K. Parida, "Some Properties of rough Sets on Fuzzy Approximation Spaces", Advances in Intelligent Systems and Computing, vol.516, 2017, pp. 179-188. doi:10.1007/978-981-10-3156-4_18.

[18] B.K.Tripathy and K.Govindarajulu: Some more properties of Covering based Multigranular rough sets, INDIA 2015, Kalyani University, J.K. Mandal et al (Eds),Information system design and applications, Advances in Intelligent Systems and Computing, 339, pp.555-564, 2015.

[19] J.T. Yao and A.V. Vasilakos, "Granular computing: perspective and Challenges". IEEE Transactions on Cybernetics, vol. 43(6), pp. 1977-1989, 2013.

[20] J. Y. Liang, F. Wang., C. Y Dang and Y.H. Qian. "An efficient rough feature selection algorithm with a multigranulation view.", International Journal of Approximate Reasoning, vol. 53, 912-926, 2012. 
[21] L.A. Zadeh, "Fuzzy Sets', Information and Control, vol. 8, pp. $338-353,1965$.

[22] L.A. Zadeh, "Fuzzy sets and information granularity", In: Gupta, M. M., R. K. Ragade and R. R. Yager eds., Advances in Fuzzy Set Theory and Applications,Vol.6.North-Holland, New York. 1979. pp. 3-18.

[23] L.A. Zadeh, "Towards a theory of fuzzy information granulation and its centrality in human reasoning and fuzzy logic", Fuzzy Sets and Systems, vol. 90(2), pp. 111$127,1997$.

[24] L.A. Zadeh, "Fuzzy Graphs, Rough Sets and Information granularity", Proceedings of Third international workshop on rough sets and soft computing. San Jose.1994, pp. 1012.

[25] M. Panda, A. Abraham and B.K.Tripathy, "Soft Granular Computing based classification using hybrid fuzzy-KNNSVM", Intelligent Decision technologies, vol.10 (2), pp.115-128, 2016.

[26] M. Nagaraju and B. K. Tripathy, "Approximate equalities for covering based Optimistic Multigranular Rough Sets and Their Properties", IIOAB Journal, vol.6(4), pp.77-97, 2015.

[27] B. K. Tripathy and R. Raghavan: On Some Topological Properties of Multigranular Rough Sets, Journal of Advances in Applied science Research, Vol.2, no.3. pp.536-543, 2011.

[28] R. Slowinski and D. Vanderpooten, "Similarity relation as a basis for Rough Approximations". In: Wang, P.P. (ed.) Advances in Machine Intelligence \& Soft-Computing, vol. 4, Duke University press, 1997, pp. 17-33.

[29] S.K. De, R. Biswas and A.R. Roy, "Rough sets on Fuzzy Approximation Spaces". The journal of Fuzzy Mathematics, vol. 11, pp. 1-15., 2003.

[30] T.Y. Lin, "Granular Computing on Binary Relations I: Data Mining and neighbourhood systems"., In: Rough sets in Knowledge Discovery, Skowron, A. and Polkowski, L. (Eds.), Springer- Verlag., 1998, pp.107-121.

[31] W. Pedrycz, "Granular Computing: The emerging paradigm", Journal of uncertain systems, vol. 1(1), pp. 38-61, 2007.

[32] W. Pedrycz and A Bargiela, "Granular Computing: A granular signature of data", IEEE Trans Systems, Man, Cybernetics B. Cybernetics, vol. 32(2), pp. 212-224, 2002.

[33] W. Pedrycz and S. M. Chen, "Granular Computing and Intelligent Systems: Design with Information Granules of Higher Order and Higher Type", New York: Springer Verlag, 2011

[34] Y.H Qian and J.Y. Liang, "Rough set method based on Multi-granulations" Proceedings of the 5th IEEE Conference on Cognitive Informatics, vol. 1, 2006, pp. $297-304$.

[35] Y.H. Qian, J.Y. Liang and C.Y. Dang, "Pessimistic rough decision", .in: Second International workshop on Rough Set Theory, Zhou Shan, China, 2010, pp.440-449.

[36] Y.Y. Yao, "Information Granulation and Rough Set Approximation", International Journal of Intelligent System, vol. 16(1), pp. 87-104, 2001.

[37] Y.Y. Yao, "On Modelling Data Mining with Granular Computing". In A. D. Williams(Ed.), Proceedings of the 25th Annual International Computer Software and Applications Conference. IEEE, 2001, pp. 638-643.

[38] Y.Y. Yao, "Three perspectives of granular computing". Proceedings of the International Forum on Theory of GrC from Rough Set Perspective, Journal of Nanchang Institute of Technology, vol. 25, pp. 16-21, 2006.
[39] Y.Y. Yao, "A unified framework of Granular Computing", In W. Pedrycz, A. Skowron \& V. Kreinovich (Eds.), Handbook of granular Computing, Wiley Publications, 2008, pp. 401-410.

[40] Y.Y. Yao, "Granular Computing: Past, Present and Future". in Proc. IEEE international conference on Granular Computing, GrC 2008, IEEE, pp. 80-85.

[41] Z. Pawlak, "Rough Sets". Int. jour. of Computer and Information Sciences, vol. 11, pp. 341-356, 1982.

[42] Z. Pawlak, "Theoretical aspects of reasoning about data", Dordrecht, Boston, London: Kluwer academic publishers, 1991.

\section{Authors' Profiles}

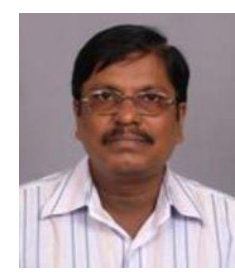

B.K. Tripathy has received 03 gold medals for topping the list of candidates at graduation and post-graduation level of Berhampur University. He was a Professor and Head of the department of Computer Science of Berhampur University till 2007. Dr. Tripathy is now working as a Senior Professor in School of Computing Science and Engineering, VIT University, Vellore, India. He has received research/academic fellowships from UGC, DST, SERC and DOE of Govt. of India for various academic pursuits. Dr. Tripathy has published more than 450 technical papers in different international journals, proceedings of reputed international conferences and edited research volumes. He has produced $27 \mathrm{PhDs}$, 13 MPhils and 4 M.S (By research) under his supervision. Dr. Tripathy has published two text books on Soft Computing and Computer Graphics and has edited 5 research volumes, besides guest editing special issues of some international journal. He was selected as honorary member of the American Mathematical Society from 1992-1994 for his distinguished contribution as a reviewer of American Mathematical Review. Dr. Tripathy has served as the member of Advisory board or Technical Programme Committee member of several International conferences inside India and abroad. Also, he has edited two research volumes for IGI publications and is editing three more research volumes. He is a life/senior member of IEEE, ACM, IRSS, CSI, ACEEE, OMS and IMS. Dr. Tripathy is an editorial board member/reviewer of more than 70 international journals. Dr. Tripathy has Technical grants for research projects from various funding agencies like UGC, DST and DRDO. His research interest includes Fuzzy Sets and Systems, Rough Sets and Knowledge Engineering, Data Clustering, Social Network Analysis, Soft Computing, Granular Computing, Content Based Learning, Neighbourhood Systems, Soft Set Theory, Social Internet of Things, Big Data Analytics, Theory of Multisets and List theory.

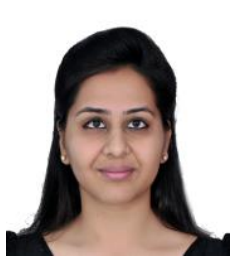

Urmi Bhambhani completed her M.Tech. in Computer Science and Engineering from VIT University. Her M.Tech Thesis was in the field of Computational Intelligence. Prior to M.Tech, she has worked for 4 years in Educational Technology, specifically looking at using Machine Learning in Education Software. 
How to cite this paper: B.K. Tripathy, Urmi Bhambhani, "Properties of Multigranular Rough Sets on Fuzzy Approximation Spaces and their Application to Rainfall Prediction", International Journal of Intelligent Systems and Applications(IJISA), Vol.10, No.11, pp.76-90, 2018. DOI: 10.5815/ijisa.2018.11.08 